\title{
Zur Entstehung des heutigen Standardlebenslaufs aus rechtshistorischer Sicht - zugleich ein Beitrag zu den juristischen Implikationen des demographischen Wandels
}

\author{
Stefan Ruppert/Kathrin Brunozzi/Christian Lange*
}

A. Einführung (Stefan Ruppert) ........ 77

I. Einleitung ...................... 77

II. Vom Recht der Lebensphasen zum

Recht des Lebenslaufs....

B. Die Definition der Kleinkindphase durch das Zusammenspiel von Staat und Gesellschaft in der öffentlichen Kleinkindererziehung

(Christian Lange) .....................

I. Einleitung .........................

II. Die Sozialisation des Kleinkindes und die Behandlung gefährdeter Kinder im frühen 19. Jahrhundert.......

III. Die öffentliche Kleinkindererziehung als schichtspezifisch die Kleinkindphase prägende Institution

92
IV. Die Entwicklung der Anstalten zur schichtübergreifenden Einrichtung. . 97

V. Fazit ........................... 98

C. Hochaltrigkeit im Recht

(Kathrin Brunozzi) ................... 99

I. Einleitung ....................... 99

II. Verrechtlichung .................. 102

III. Verrechtlichung des „Vierten Lebensalters" “...................... 104

IV. Viertes Alter und Recht? ........... 107

1. Heimrecht.................... 107

2. Betreuungsrecht................ 107

V. Schluss ............................ 108

D. Resumée (Stefan Ruppert) ............. 109

\section{A. Einführung (Stefan Ruppert)}

\section{Einleitung}

Der demographische Wandel ist allgegenwärtig. ${ }^{1}$ Wer ihn thematisiert und in einen spezifischen Kontext setzt, der erhöht seine Chancen beachtet zu werden. Elterngeld, Vereinbarkeit von Familie und Beruf, Frauenquote, Rente mit 67, Einwanderung qualifizierter Fachkräfte, steigende Krankenkassenbeiträge, Neugestaltung der sozialen Infrastruktur und Pflegekräftemangel - wer darüber mit medialem Erfolg debattieren will, der leite seine Forderungen aus Zahlen zu Geburtenraten und Lebenserwartung ab und berufe sich auf den demographischen Wandel. Nicht selten wird dann ein Bedrohungsszenario bemüht, um gesetzliche Veränderungen zu legitimieren. Die gewählten Worte zeugen meist von einer emotionalisierten Debatte. Schnell ist vom „demographischen Defizit“ die Rede. ${ }^{2}$ Durch den Begriff der Überalterung werden Menschen mit höherem Lebensalter abgewertet. ${ }^{3}$ Den eindringlichen Forderungen nach Veränderungsbedarf ist nicht selten ein negatives Altersbild immanent.

* Dr. Stefan Ruppert leitet seit 2005 die Forschungsgruppe „Lebensalter und Recht“ am MPI für europäische Rechtsgeschichte in Frankfurt am Main. Seit 2009 ist er Mitglied des Deutschen Bundestags. Dr. Kathrin Brunozzi und Christian Lange waren wissenschaftliche Mitarbeiter in der Forschergruppe und sind heute als Richterin bzw. Referent einer Bundestagsfraktion tätig.

1 So ergibt eine Suche zu dem Begriff bei Google mehr als 1,9 Millionen Treffer aus den unterschiedlichsten Bereichen.

2 Hans-Werner Sinn, Das demographische Defizit - die Fakten, die Folgen, die Ursachen und die Politikimplikationen, in: ifo Schnelldienst 5/2003, S. 20-36.

3 Dieser allgemeinen Einschätzung wird auch immer wieder entgegengetreten, vgl. dazu jüngst Axel Börsch-Supan, Unsere gewonnenen Jahre, in: FAZ vom 25.2.2011, S. 11. 
Die Skala düsterer Assoziationen endet beim Beschwören des „Aussterbens der Deutschen“, das Vereine wie den „Schutzbund für das Deutsche Volk e.V.“ zu rechtsradikalen Forderungen veranlasst. ${ }^{4}$ Bemerkenswert in den zahlreichen Diskussionen ist die Dominanz ökonomischer Zwänge und Argumentationsmuster.

An dieser Stelle soll keinesfalls negiert werden, dass die sich verändernde Altersstruktur der deutschen Gesellschaft eben diese Gesellschaft vor große Herausforderungen stellt. Sie werden aber immer wieder überschätzt. ${ }^{5}$ Eine in diesem politischen Kontext erhobene Forderung ist die nach der Veränderung unseres modernen Lebenslaufmodells. Durch einen früheren Einstieg in die Erwerbstätigkeit und ihre Verlängerung im Alter will man die drohenden Produktivitätsverluste einer alternden Gesellschaft kompensieren. Der Wunsch nach Veränderung des normalen Lebenslaufs lässt die Frage naheliegend erscheinen, wie diese Lebenslaufstruktur eigentlich entstanden ist. Ihrer Beantwortung nähert sich dieser Beitrag. Es soll gezeigt werden, welchen Beitrag rechtliche Regelungen an der Entstehung eines modernen Standardlebenslaufs haben. Da es um eine umfassende Darstellung nicht gehen kann, wählen wir einen Weg aus der Kombination allgemeiner Bemerkungen zur Wirkungsweise des Rechts bei der Herausbildung einer modernen Lebenslaufstruktur und verbinden ihn mit zwei rechtshistorischen Detailstudien, die beispielhaft die Entwicklung in Teilbereichen nachzeichnen.

Es geht uns um einen Beitrag für eine gründlichere, sachlichere und weniger emotionalisierte Analyse des modernen Lebenslaufmodells. Die Vielzahl wirtschaftlicher, soziologischer und politikwissenschaftlicher Betrachtungsweisen soll um einen juristischen Beitrag ergänzt werden. Es gilt, die normativen Implikationen der Entstehung des modernen Standardlebenslaufs genauer zu betrachten. Drei Aspekte der demographischen Debatte sind auch für den rechtlich strukturierten Lebenslauf bedeutsam:

1. Zunehmend wird die Frage thematisiert, ob man dem demographischen Wandel durch neue rechtliche und institutionelle Rahmenbedingungen entgegen wirken kann. Das Elterngeld etwa wird als ein Mittel des Gesetzgebers propagiert, mit gesetzgeberischen Mitteln die Fertilität zu steigern. ${ }^{6}$ An dieser Diskussion beteiligen sich Juristen nur am Rande, sie wird stärker von Demographen und Soziologen ge-

4 Der Schutzbund für das Deutsche Volk e. V. verbindet seine bevölkerungspolitischen Forderungen mit einem streng nationalistischen und politisch auf der extremen Rechten angesiedelten Konzept sowie politischen Forderungen, den Beitritt der Türkei und verstärkte Einwanderung zu unterbinden, näheres unter www.schutzbund.de.

5 Vgl. hierzu etwa die sachliche Einordnung von Sergei Scherbov/Warren Sanderson, Negative Folgen der Alterung bislang überbewertet. Neue Maßzahlen für aktuelle Bevölkerungsentwicklung, in: Demografische Forschung aus erster Hand, Heft 4/2010, S. 1-2.

6 Vgl. dazu den umfassenden Ansatz von Hans Bertram, Nachhaltige Familienpolitik für alle Generationen-Zeit, Infrastruktur und Geld, in: Ursula von der Leyen (Hrsg.), Füreinander da sein. Miteinander handeln. Warum die Generationen sich gegenseitig brauchen, Berlin 2007, S. 107-128. 
führt. Dabei wird das Wort der „Bevölkerungspolitik“ wegen der historischen Belastung vermieden. ${ }^{7}$

2. Durch den demographischen Wandel verändert sich das Verhältnis der Generationen untereinander. Die Metapher vom Generationenvertrag, ${ }^{8}$ der einerseits immer wieder neu geschlossen werden soll, dessen Vertragspartner aber zunehmend in eine ungleiche Verhandlungsposition zu kommen drohen, weist mittels der Annahme eines fiktiven Vertragsschlusses auf diesen juristischen Aspekt hin. Heute drehen sich viele Diskurse zumeist um die Frage, wie weniger Arbeitnehmer die Altersversorgung einer wachsenden Zahl von Rentnern sichern können. ${ }^{9}$ Entsprechend widmen sich zahlreiche juristische Beiträge dem Recht der Rentenversicherung in der alternden Gesellschaft ${ }^{10}$ sowie den rechtlichen Rahmenbedingungen für die an Bedeutung gewinnende private Altersvorsorge. ${ }^{11}$ Auch diese Diskussion unter Juristen ist älter als zumeist angenommen wird. ${ }^{12}$

3. Der demographische Wandel rückt die alten Menschen in den Mittelpunkt auch der juristischen Debatten. Ihre Zahl wird sichtbar größer. Ihr Anteil an der Gesamtbevölkerung steigt noch stärker. Das Alter hat in gewisser Weise Konjunktur und das lässt die Frage naheliegend erscheinen, ob es eines „Rechts der Älteren“13 bedürfe. ${ }^{14}$ Die deutsche Rechtswissenschaft rezipiert dabei vor allem Vorbilder aus den USA. ${ }^{15}$ Juristen thematisieren die alternde Gesellschaft unter anderem als Verfas-

7 Vgl. dazu Rainer Mackensen, Bevölkerungslehre und Bevölkerungspolitik im „Dritten Reich“, Opladen 2004.

8 Vgl. hierzu etwa Peter Häberle, Ein Verfassungsrecht für künftige Generationen - die „andere“ Form des Gesellschaftsvertrages: der Generationenvertrag, in: Franz Ruland (Hrsg.), Verfassung, Theorie und Praxis des Sozialstaats. Festschrift für Hans F. Zacher zum 70. Geburtstag, Heidelberg 1998, S. 215-233; auch der Generationenvertrag wird oft stark aus der Sicht der Sozial- und Wirtschaftsgeschichte betrachtet, vgl. dazu etwa Gerd Hardach, Der Generationenvertrag. Lebenslauf und Lebenseinkommen in Deutschland in zwei Jahrhunderten, Berlin 2006.

9 Vgl. hierzu etwa Timo Hebeler, Generationengerechtigkeit als verfassungsrechtliches Gebot in der sozialen Rentenversicherung, Baden-Baden 2001.

10 Eine umfassende Darstellung aller rechtlichen Probleme findet sich bei Eberhard Eichenhofer (Hrsg.), Handbuch der gesetzlichen Rentenversicherung - SGB VI. Köln 2011; einer systematischen wissenschaftlichen Bearbeitung des Themas widmet sich etwa das „Forschungsnetzwerk Alterssicherung“ der Deutschen Rentenversicherung, näheres unter www.fna-rv.de.

11 Vgl. hierzu die umfassende Studie von Markus Roth, Private Altersvorsorge: Betriebsrentenrecht und individuelle Vorsorge : Eine rechtsvergleichende Gesamtschau, Tübingen 2009.

12 Vgl. hierzu Thomas Duve, Generationengerechtigkeit und Altersversorgung in der juristischen Literatur zur Rechtsstellung alter Menschen des 17. und frühen 18. Jahrhunderts, in: Stefan Brakensiek/ Heide Wunder/Michael Stolleis (Hrsg.): Generationengerechtigkeit? Normen und Praxis im Ehe und Ehegüterrecht 1500-1850, Berlin 2006, S. 45-62.

13 Vgl. etwa aus neuerer Zeit Gerhard Igl/Thomas Klie (Hrsg.), Das Recht der älteren Menschen, BadenBaden 2007; ein frühes Beispiel ist etwa Hans Hartmann von Schlotheim, Recht im Alter. Kleine Handreichung für die Altenarbeit, Kassel 1978.

14 Gerhard Igl, Das „Recht der älteren Menschen“ - Ist es wünschenswert, für die älteren Menschen besondere rechtliche Vorkehrungen zu treffen?, in: Zeitschrift für Gerontologie 1990, S. 62-67.

15 Vgl. hierzu Lawrence A. Frolik, The Developing Field of Elder Law. A historical Perspective, in: The Elder Law Journal 1993, Vol 1, number 1, S.1-18; ders., The Developing Field of Elder Law Redux: Ten Years After, in: The Elder Law Journal 2002, Vol 10, number 1, S. 1-14. 
sungsproblem oder behandeln zumindest die Rechtsstellung älterer Menschen in Verfassungen. ${ }^{16}$ Rechtsanwaltskanzleien entdecken neue Mandanten unter den Älteren. ${ }^{17}$

Der moderne Standardlebenslauf besteht aus den Lebensphasen Jugend, Erwerbsbiographie und Alter. Jede dieser Lebensphasen ist wiederum durch eine Vielzahl von Normen binnenstrukturiert. Recht ordnet den Übergang von der arbeitsfreien Jugend in das Erwerbsleben und von dort in die erneut arbeitsfreie Rente. Für diesen Beitrag haben wir uns entschieden, gerade nicht die Übergänge zwischen den Lebensphasen in den Blick zu nehmen, sondern nach diesen allgemeinen einleitenden Bemerkungen anhand von zwei Beispielen die weitere Unterteilung und Binnenstrukturierung zweier Lebensphasen zu betrachten.

Immer wieder wird darauf hingewiesen, dass sich die Lebenslaufstruktur in modernen Gesellschaften massiv geändert hat und weiter ändern muss. Menschen durchlaufen eine immer längere Ausbildung, Paare heiraten später und entschließen sich auch später dazu eine Familie zu gründen. Aus der Familiengeschichte wissen wir, dass diese Muster sich auch in früheren Gesellschaften gewandelt haben. ${ }^{18}$ Gleichwohl knüpfen viele Maßnahmen, die Symptome oder Ursachen des demographischen Wandels begegnen wollen, genau an dieser Lebenslaufstruktur an. Die vielfach gepredigten Maßnahmen zur besseren Vereinbarkeit von Familie und Beruf versuchen eben die veränderte Lebenslaufstruktur mit längerer Ausbildungszeit und höheren Beschäftigungsraten hochqualifizierter Frauen mit dem Wunsch nach einer Familiengründung in Einklang zu bringen. Die Debatten um die Erhöhung des Rentenalters und der Verkürzung von Schul- und Studienzeiten sind darauf gerichtet, die Länge der als volkswirtschaftlich produktiv verstandenen Erwerbsbiographie gegenüber den eher arbeitsfrei konzipierten Lebensphasen Jugend und Ruhestand wieder auszudehnen.

Allerdings fällt auf, welche Schwierigkeiten bei der Umsetzung einiger grundlegender Reformen entstehen. Die Gegenwehr gegen die Rente mit 67 ist sicherlich der prominenteste Beispielsfall. Bei genauerer Betrachtung stellt sich aber heraus, dass es mit der Veränderung einer Altersgrenze alleine nicht getan ist. Zu komplex ist das $\mathrm{Zu}$ -

16 Vgl. Peter Häberle, Altern und Alter des Menschen als Verfassungsproblem, in: Peter Badura/Rupert Scholz (Hrsg.), Wege und Verfahren des Verfassungslebens. Festschrift für Peter Lerche zum 65. Geburtstag, München 1993, S. 189-211.

17 So etwa die Rechtsanwaltskanzlei Georg Zenker in Berlin vgl. hierzu http://www.kanzlei-seniorenrecht.de/; ebenso die Kanzlei Dr. Lang und Kollegen in München, vgl. hierzu http:/www.anwaltskanzlei-muenchen.de/seniorenrecht/, vgl. auch Ronald Richter/Bettina Schmidt/Michael Klatt/Gudrun Doering-Striening (Hrsg), Seniorenrecht in der anwaltlichen Praxis, Baden-Baden 2006.

18 Einen guten Überblick gibt Tamara K. Hareven, Familie, Lebenslauf und Sozialgeschichte, in: Josef Ehmer/dies./Richard Wall (Hrsg.), Historische Familienforschung. Ergebnisse und Kontroversen. Michael Mitterauer zum 60. Geburtstag, Frankfurt am Main 1997, S. 17-37. 
sammenspiel vieler Normen und zu sehr ist eine Lebensphase wie etwa die Jugend ihrerseits in aufeinander abgestimmte Lebensphasen unterteilt.

Bevor man zur eigentlichen Entstehung des juristischen Lebenslaufmodells kommen kann noch einige Worte zum Forschungsstand. Die Lebenslaufforschung wird von Soziologen, Demographen und Historikern dominiert. Schaut man bei juristischen Autoren nach Vorgängern für die Erforschung des Themas, dann finden sich vor allem Stimmen zum Recht einzelner Lebensphasen. Insbesondere die juristische Sonderrechtsliteratur des 17. und 18. Jahrhunderts systematisierte mehrfach die diversen Sonderrechte älterer Menschen. ${ }^{19}$ Im 19. und bis zum ausgehenden 20. Jahrhundert hatten diese „Altenrechte“ kaum Nachfolger gefunden. Demgegenüber gab es in der ersten Hälfte des 20. Jahrhunderts immer wieder Versuche, das „Jugendrecht" als eigene rechtswissenschaftliche Disziplin zu etablieren. So erschien seit den Zwanzigerjahren ein „Jahrbuch des Jugendrechts“. ${ }^{20}$ Mittlerweile scheinen diese Versuche, die damals Strafrecht, Jugendhilferecht, aber auch Teile des Zivilrechts umfassten, gescheitert. ${ }^{21}$ Über die Gründe soll hier nicht weiter spekuliert werden. Festgehalten sei aber, dass politische Diskurse über die Bedeutung der Jugend in der ersten Hälfte des 20. Jahrhunderts die entsprechenden juristischen Veröffentlichungen genauso stimuliert haben dürften, wie die alternde Gesellschaft und der demographische Wandel die Initiativen zum „Recht der Älteren“ es heute tun.

In den letzten Jahren mehren sich die Versuche, ein „Recht der Älteren“ auch in der deutschen rechtswissenschaftlichen Debatte zu etablieren. Es handelt sich um zumeist an der Praxis orientierte juristische Ratgeberliteratur. Thematisch ging es den Autoren um Hinweise zu Fragen der Patientenverfügung, der Errichtung des Testaments, aber auch um die Betonung der Rechte älterer Menschen. ${ }^{22}$ An juristischen Fakultäten fanden erste Seminare zum „Altenrecht“ ${ }^{23}$ statt und es entstanden Rechtsanwaltskanzleien mit dem Tätigkeitsschwerpunkt Seniorenrecht. ${ }^{24}$ Sie spezialisieren

$19 \mathrm{Zu}$ einem frühen Versuch aus dem Jahr 1705 von Theodosius Schoepffer vgl. Arnold Becker/Marc Laureys/Karl August Neuhausen/Georg Rudinger(Hrsg.), Theodosius Schoepffers „Gerontologia seu Tractatus de hure senum“. Kulturwissenschaftliche Studien zu einem vergessenen Traktat über das Altenrecht, Bonn 2011, S. 205-244; Nachweise finden sich umfassend bei Thomas Duve, Sonderrecht in der Frühen Neuzeit - Studien zum ius singular und den privilegia miserabilium personarum, senum und indorum in Alter und Neuer Welt, Frankfurt am Main 2008, S. 205.

20 Das Jahrbuch wurde seit 1922 und bis 1943 zunächst von Karl Hagemann und später von Heinrich Webler herausgegeben. Nach dem 2. Weltkrieg erschien als Nachfolgepublikation noch das Archiv für Jugendrecht seit 1952, dieses wurde aber 1958 ebenfalls eingestellt.

21 Der vorerst letzte Versuch dieses übergreifend darzustellen stammt von Thilo Ramm, Jugendrecht. Ein Lehrbuch, München 1990.

22 Vgl. dazu den interessanten Sammelband von Gerhard Igl/Thomas Klie (Hrsg.), Das Recht der älteren Menschen, Baden-Baden 2007.

23 So etwa an der Universität Innsbruck vgl. hierzu die Darstellung im Internet unter http:// www.uibk.ac.at/zivilrecht/mitarbeiter/ganner/altenrecht.html.

24 So etwa die Rechtsanwaltskanzlei Georg Zenker in Berlin vgl. hierzu http://www.kanzlei-seniorenrecht.de/; ebenso die Kanzlei Dr. Lang und Kollegen in München, vgl. hierzu http://www.anwaltskanzlei-muenchen.de/seniorenrecht/. 
sich auf „rechtliche Probleme, die sich typischerweise im fortgeschrittenen Alter stellen“ mit dem Ziel „eine möglichst selbstbestimmte Lebensplanung im Alter zu ermöglichen “. ${ }^{25}$ Eine Initiative von Juristen der Max-Planck-Gesellschaft gibt gerade ein eher wissenschaftlich orientiertes Handbuch zum „Recht der Älteren“ heraus. ${ }^{26}$ Unter den Vorzeichen der aktuellen Debatten über demographischen Wandel und der Alterung der Gesellschaft entsteht der Wunsch nach einer Systematisierung des geltenden Rechts unter der Kategorie des Lebensalters. Die Zukunft eines eigenen Rechtsgebiets, des „Rechts der Älteren“, erscheint angesichts der in Deutschland eher anders verlaufenden Prozesse der Fächerbildung offen. ${ }^{27}$ Es bedürfte zur Festigung des Fachs einer gründlichen Debatte über die Form der Systematisierung von „Altenrecht“". Sollen einzelne, für ältere Menschen besonders relevante Rechtsgebiete wie das Heimrecht, das Betreuungsrecht, das Recht der Pflege- und Rentenversicherung oder das Recht gegen Altersdiskriminierung im Vordergrund stehen? Oder soll man einen weiteren Ansatz wählen, der zusätzlich in allen Rechtsgebieten besondere Aspekte, wie etwa die Lehre der Strafzumessung für alte Menschen oder ihren besonderen Schutz im durch technischen Wandel veränderten und beschleunigten Geschäftsverkehr des Internets, herausstellt?

\section{Vom Recht der Lebensphasen zum Recht des Lebenslaufs}

Wie nun ist das normative Standardlebenslaufmuster unserer Tage entstanden? Jede Lebensphase ist durch Altersgrenzen oder altersspezifische Regelungen definiert. Dabei wird insbesondere die Jugend nicht durch "die“ eine Altersgrenze beendet. Bei aller Bedeutung der Volljährigkeit spielten im männlichen Lebenslauf die Absolvierung der Wehrpflicht oder allgemein die Beendigung der Ausbildung eine ebenfalls wichtige Rolle. Reiht man die Rechte der einzelnen Lebensphasen aneinander, indem einem „Jugendrecht“ ein „Recht der Erwachsenen“ oder vielleicht etwas spezifischer ein „Recht der Erwerbstätigen“ und ein „Recht der Älteren“ folgt, dann entsteht ein juristisch konturiertes Lebenslaufmodell. Der von den Sozialwissenschaften demgegenüber gewählte Ansatz bei der Untersuchung des menschlichen Lebenslaufs ist gerade nicht normativ. Anhand von empirischen Daten deckten historisch arbeitende

25 So nach eigener Aussage die Kanzlei „Menschen und Rechte“, vgl. hierzu http://www.menschenundrechte.de/rechtsgebiete,5,seniorenrecht.php.

26 Getragen wird die Initiative von Prof. Dr. Ulrich Becker vom MPI für Sozialrecht in München und Prof. Dr. Markus Roth (Marburg) unter Beteiligung des Autors. Das Handbuch soll Anfang 2012 erscheinen.

27 Die Gedanken der letzten Sätze finden sich bereits in einem Heidelberger Akademievortrag, der demnächst erscheinen wird. 
Demographen ${ }^{28}$ und Lebenslaufsoziologen ${ }^{29}$ für die Moderne relativ stabile Lebenslaufmuster auf. Hier wird das Anliegen vertreten, den Anteil des Rechts an der Entstehung dieser Strukturen zu betonen. Es soll gezeigt werden, dass es eben nicht nur die individuelle Einzelentscheidung einer großen Zahl von Individuen war, die sich entschlossen zu heiraten, das elterliche Haus zu verlassen oder ihren Hof oder Handwerksbetrieb an die nächste Generation zu übergeben, ${ }^{30}$ die das moderne Lebenslaufmuster prägten. Die moderne Lebenslaufstruktur verdankt sich auch keinesfalls nur wirtschaftlichen Entwicklungen oder ist gar eine anthropologische Konstante. Vielmehr strukturiert eine Vielzahl von Normen unseren Lebenslauf, die Regelungen räumen Rechte und Pflichten ein. Viele richten sich etwa bei der Geschäftsfähigkeit oder der Strafmündigkeit an alle. Andere sanktionieren nur diejenigen, die den normativ erwarteten Idealverlauf verlassen haben wie etwa das Jugendstrafrecht. Wieder andere setzen lediglich Anreize, eine gewisse biographische Entscheidung in einem spezifischen Alter zu treffen. ${ }^{31}$

Der sogenannte institutionalisierte Lebenslauf ist auch aus juristischer Sicht dreigeteilt. Das aber beschreibt nur eine grob vereinfachende Struktur und verhindert, dass man die komplexe normative Strukturierung jeder einzelnen Lebensphase besser versteht. Die Lebensphase Jugend wurde in den letzten 200 Jahren zunehmend ausgedehnt und arbeitsfrei konzipiert. ${ }^{32}$ Die Durchsetzung der Schulpflicht zu Beginn des 19. Jahrhunderts leistete den wesentlichen Beitrag zur Verlängerung der Kindheit. Bereits zuvor war aber durchaus auch die Kleinkindphase nicht frei von staatlicher Gesetzgebung, wie noch zu zeigen sein wird. Flankiert wurde die Schulgesetzgebung durch Fabrikarbeitsverbote für Kinder und Jugendliche. Höhere Bildungsabschlüsse und die berufliche Ausbildung wurden rechtlich konturiert. Auch die

28 Vgl. zur historischen Demographie Arthur E. Imhof, Einführung in die Historische Demographie, München 1977; ders., Die Zunahme unserer Lebensspanne seit 300 Jahren und ihre Folgen, Stuttgart 1996; einen Überblick über Forschungsstand und Gegenstand geben Josef Ehmer, Bevölkerungsgeschichte und historische Demographie, München 2004; analog zur älteren Zeit Christian Pfister, Bevölkerungsgeschichte und historische Demographie 1500-1800, München 1994 jeweils m. w. N.

29 Vgl. hierzu Martin Kohli, Die Institutionalisierung des Lebenslaufs. Historische Befunde und theoretische Argumente, in: Kölner Zeitschrift für Soziologie und Sozialpsychologie 1985, S. 1-29; ders., Der institutionalisierte Lebenslauf: ein Blick zurück und nach vorn, in: Jutta Allmendinger (Hrsg.), Entstaatlichung und Soziale Sicherheit. Verhandlungen des 31. Kongresses der Deutschen Gesellschaft für Soziologie in Leipzig 2002 Bd. 1, Opladen 2003, S. 525-545; einen näher an juristischen Kategorien liegenden Ansatz verfolgen Karl Ulrich Mayer/Urs Schoepflin, The state and the life course, in: Annual Review of Sociology 1989, 187-209; aus neuerer Zeit vgl. ders., New Trends in Life Course Research, in: Annual Review of Sociology Bd. 35 (2009), S. 493-514 m w. N.

30 Vgl. zu der Wechselwirkung von den Lebenslauf strukturierenden Gesetzen und der familiären Entscheidung, den Hof zu übergeben insbesondere Birgit Fastenmayer, Hofübergabe als Altersversorgung. Generationenwechsel in der Landwirtschaft 1870-1957, Frankfurt am Main 2009.

31 Das gilt etwa für die Übergabe landwirtschaftlicher Höfe seit der Einführung einer Altersversorgung für Landwirte 1957, vgl. hierzu Birgit Fastenmayer, Hofübergabe (Fn. 30), S. 265-279.

32 Vgl. hierzu Stefan Ruppert, Neues „Jugendrecht“ und Fabrikschutzgesetzgebung im Vormärz. Zur Bedeutung von Normativität für die Entstehung der Lebensphase Jugend, in: Jahrbuch für Wirtschaftsgeschichte Bd. 1, Berlin 2008, S. 55-75. 
Entstehung des Jugendstrafrechts, das Jugendliche nun nicht mehr nur milder, sondern schlicht anders und auf Resozialisierung ausgerichtet bestrafen wollte, liegt im 19. Jahrhundert. ${ }^{33}$ Es entstand ein Jugendfürsorgerecht, das Wahlalter wurde sukzessive gesenkt und durch die Einführung der allgemeinen Wehrpflicht wurde die männliche Jugend beendet. ${ }^{34}$ Das Zusammenwirken einer Vielzahl von Normen schuf die Lebensphase Jugend neu.

Kindheit und Jugend folgt seit dem ausgehenden 19. Jahrhundert die stark an sozialversicherungspflichtige Beschäftigungsverhältnisse anknüpfende Erwerbsbiographie. Diese zunächst von abhängig beschäftigten Männern geprägte Lebensphase bestimmte im Laufe der zweiten Hälfte des 20. Jahrhunderts zunehmend auch den weiblichen Lebenslauf. ${ }^{35}$ Zudem richteten sich etwa die freien Berufe mit ihren am Vorbild der Rentenversicherung orientierten Versorgungswerken ebenfalls an diesem Muster aus. Mit dem Wandel der zunächst vorrangig auf die Absicherung von Invalidität und einer kleinen Zusatzversorgung im Alter abzielenden Rentenversicherung zu einer vollwertigen Altersversorgung spätestens seit der Adenauerschen Rentenreform von $1957^{36}$ ist der Ruhestand als dritte Lebensphase klarer von der Erwerbsbiographie getrennt worden. Entstanden ist damit eine Lebensphase, die ebenfalls weitgehend arbeitsfrei konzipiert ist. ${ }^{37}$ Mittlerweile, und davon wird noch die Rede sein, bildet sich so etwas wie eine vierte Lebensphase heraus. Anders als das ihr vorausgehende dritte Alter, das aktiv teilhabend gedacht wird, ist das vierte Lebensalter von Pflegebedürftigkeit geprägt. ${ }^{38}$

Bereits diese Kurzvorstellung des dreigeteilten Lebenslaufs macht deutlich, dass Normativität für die Entstehung unseres Lebenslaufmodells eine entscheidende Rolle spielt. Recht in der Form von Altersgrenzen oder altersspezifischen Regelungen wie den Anwartschaften in der Rentenversicherung segmentiert den menschlichen Le-

33 Vgl. zu der Entstehung des Jugendstrafrechts insbesondere in Russland mit Hinweisen auf die deutsche Entwicklung Tatjana Mill, Zur Erziehung verurteilt. Die Entwicklung des Jugendstrafrechts im zaristischen Russland, Frankfurt am Main 2010; zur Entwicklung in Deutschland vgl. Markus Fritsch, Die jugendstrafrechtliche Reformbewegung (1871-1923). Die Entwicklung bis zum ersten Jugendgerichtsgesetz unter besonderer Berücksichtigung der Diskussion über die Altersgrenzen der Strafmündigkeit, Freiburg i. Br. 1999.

34 Vgl. zur Entstehung der Jugend aus rechtshistorischer Sicht demnächst Stefan Ruppert, Recht macht jung - zur Entstehung der Jugend aus rechtshistorischer Sicht, voraussichtlich Frankfurt am Main 2012.

35 Vgl. dazu Dorothea Noll, „... ohne Hoffnung im Alter jemals auch nur einen Pfennig Rente zu erhalten...". Die Geschichte der weiblichen Erwerbsbiographie in der gesetzlichen Rentenversicherung, Frankfurt am Main 2010.

36 Vgl. hierzu den kurzen Überblick bei Michael Stolleis, Geschichte des Sozialrechts in Deutschland. Ein Grundriß, Stuttgart 2003, S. 275-279; Hans Günter Hockerts, Konrad Adenauer und die Rentenreform von 1957, in: Konrad Repgen (Hrsg.), Die dynamische Rente in der Ära Adenauer und heute, Stuttgart 1978, S. 11-30.

37 Vgl. hierzu Christoph Conrad. Vom Greis zum Rentner. Der Strukturwandel des Alters in Deutschland von 1830 bis 1930, Göttingen 1994.

38 Vgl. dazu unten die Ausführungen von Kathrin Brunozzi. 
benslauf. Maßgeblich haben Normen zur Entstehung, aber auch zur Stabilisierung und Perpetuierung des institutionalisierten Lebenslaufs beigetragen. ${ }^{39}$ Es ist wichtig dies zu sehen, weil das einmal entstandene normative Lebenslaufmodell so leicht nicht veränderbar ist. Viele Altersgrenzen sind tief im kollektiven Bewusstsein verwurzelt, sie geben den Menschen Orientierung und schaffen Rechtspositionen, die stark von der Perspektive der Erlangung einer vorteilhaften Rechtsposition geprägt sind. Später soll am Beispiel zweier Binnenstrukturierungen der Lebensphasen - der Strukturierung der Kleinkindphase und des hohen Alters - gezeigt werden, dass ein fein gesponnenes normatives Netz dem tradierten Lebenslaufmodell eine haltbare innere Struktur verleiht.

Allerdings scheint es mächtig zu krachen im Gebälk des institutionalisierten Lebenslaufmodells. Vorrangig gerät nicht seine dreigeteilte Struktur unter Druck, sondern vor allem das zeitliche Verhältnis der einzelnen Phasen zueinander. Am sichtbarsten ist dies bei der Anhebung der Renteneintrittsgrenze, aber auch die Jugend wird durch kürzere Schulzeiten bis zum Abitur, die abgeschaffte Wehrpflicht und die Aufwertung des Bachelors weniger lang. Die politischen Schwierigkeiten für solche Eingriffe sind allgemein bekannt. Die Verkürzung der maximalen Dauer der Jugendphase erscheint vielen als unwillkommene Verdichtung von Lebenszeit. ${ }^{40}$ Was der Gesetzgeber als Leerlauf auf Kosten der Rentenkassen einstuft, betrachtet der Betroffene als Verlust von biographischer Gestaltungsfreiheit. Die Verlängerung der Lebensarbeitszeit begegnet bekanntlich noch höheren Widerständen.

Unterstellt man einmal die Richtigkeit der These vom relativ stabilen dreigeteilten Lebenslauf, so liegt die Frage nach Erklärungen hierfür nahe. Neben der bereits erwähnten engmaschigen Binnenstrukturierung sind dafür weitere Gründe anzuführen. Zunächst beobachten wir eine fortwährende Verrechtlichung aller Lebensphasen. Die altersadäquate rechtliche Adressierung reicht von der pränatalen Phase bis ins höchste Alter. Zudem lassen sich nur schwer isolierte gesetzgeberische Maßnahmen treffen, weil dieses Drehen an einer Stellschraube weitreichende Auswirkungen auf andere Regelungen hat. Die Schulzeit, die Ausbildung, Geschäfts-, Religions- und Strafmündigkeit, aber auch der Mutterschutz oder die Elternzeit, Anwartschaften in sozialen Sicherungssystemen - all diese juristischen Lebensphasenmodelle sind aufeinander bezogen. Die normative Lebenslaufstruktur von den ersten Lebensjahren bis zum Tod ist zudem in hohem Maße kulturell akzeptiert. Sie wird seit Generatio-

39 Vgl. zu den Bedingungen der Entstehung der heutigen normativen Lebenslaufstruktur Stefan Ruppert, Lebensalter und Recht. Zur Segmentierung des menschlichen Lebenslaufs durch rechtliche Regelungen seit 1750 im gleichnamigen Tagungsband, Frankfurt am Main 2010, S. VII-XXIII. In dem Band finden sich darüber hinaus zahlreiche Beispiele für die Segmentierung des menschlichen Lebenslaufs in Lebensphasen.

40 Vgl. etwa die Aktivitäten der Aktionsgemeinschaft Bildungsstreik 2009, einem Zusammenschluss von nach eigenen Angaben mehr als 200 Schüler- und Studentenorganisationen unter http://www.bildungsstreik.net. Eingesehen am 22.6.2011. 
nen eingeübt und als adäquat betrachtet. Zudem sind viele Altersgrenzen das Resultat eingehender Aushandlungsprozesse. Nicht von ungefähr wird um wenige juristische Regelungen so sehr gerungen wie um das Wahlalter, die Geschäftsfähigkeit oder etwas profaner das Mindestalter zum Erwerb eines Führerscheins oder die Rentengrenze. Menschen haben an diesen Zäsuren mehr als nur politische oder materielle Interessen, sie orientieren sich an ihnen. Wenn die eigene Biographieplanung eine der wichtigsten, aber auch schwierigsten Aufgaben für den modernen Menschen darstellt, dann benötigt er normative Leitplanken für diese Planung. Die Versetzung in die nächste Klasse, die Planung bis zum Ende der Schulzeit oder des Studiums strukturieren den eigenen biographischen Horizont genauso wie die Altersgrenze zum Eintritt in den Ruhestand. Rückblickend erscheint zudem der Übergang von einer normativ strukturierten Lebensphase in die nächste vielen Menschen als angemessen. Das wird deutlich, wenn Erwachsene sich mehrheitlich gegen die Senkung des Wahlalters wenden. Gerade weil das tradierte Lebenslaufmuster weithin akzeptiert ist und die Tendenz eher zu einer Verrechtlichung aller Lebensphasen, ja sogar des Sterbens und der Präimplantationsdiagnostik geht, stehen einer Destandardisierung eine Vielzahl von Normen entgegen.

Im Folgenden soll durch zwei detailliertere Darstellungen der normativen Entstehung von Teillebensphasen versucht werden, diese Annahmen am rechtshistorischen Quellenmaterial zu verdeutlichen. Dabei wird deutlich, dass der dreigeteilte rechtlich strukturierte Lebenslauf in vielen Variationen eines Themas existiert. Manche Normen gelten nur für ein Geschlecht oder eine besondere Personengruppe, andere wiederum betreffen Menschen nur in bestimmten Lebenssituationen. Wir haben bewusst den Beginn und das Ende des Lebens für diese Beispiele ausgewählt. Zum einen umspannen diese Beispiele dann den gesamten Lebenslauf. Sie machen deutlich, dass jenseits der immer wieder erwähnten Dreiteilung eine deutlich weitergehende normative Strukturierung des menschlichen Lebenslaufs erfolgt. Sie decken aber auch in historischer Hinsicht fast den gesamten Entstehungszeitraum des normativen Standardlebenslaufs ab. Während sich die Strukturierung der Kleinkindphase bereits früh im 19. Jahrhundert herauszubilden beginnt, ist die Entstehung des „Vierten Alters“ im Recht ein Resultat der juristischen Zeitgeschichte und noch nicht abgeschlossen. 


\section{B. Die Definition der Kleinkindphase durch das Zusammenspiel von Staat und Gesellschaft in der öffentlichen Kleinkindererziehung (Christian Lange)}

\section{Einleitung}

Die Erziehung kleiner Kinder ist in Deutschland nicht nur eine familiäre Aufgabe. 92,2 \% der Kinder im Alter von drei bis sechs Jahren wurden 2010 tagsüber in Kindergärten oder von Tagesmüttern bzw. -vätern betreut. ${ }^{41}$ In der Zahl drücken sich Erfolg und gesellschaftliche Akzeptanz dieser Einrichtungen aus. Deutlich wird die wichtige Rolle, die dem außerfamiliären Erziehungs- und Bildungssystem schon vor der Einschulung bei der Entwicklung der Kinder zukommt. Die Stunden in Kindergärten prägen die drei- bis sechsjährigen Kinder. In der gesellschaftlichen Betrachtung, aber auch in der persönlichen Rückschau verbindet man diesen Lebensabschnitt mit der Einrichtung.

Die Bereitstellung außerfamiliärer Betreuungsmöglichkeiten wird dabei als gesellschaftliche und als staatliche Aufgabe angesehen. Neben dem freiwilligen Engagement von Elterninitiativen und freien Trägern, wie der Caritas, der Diakonie oder der Arbeiterwohlfahrt, nehmen sich auch der Bund, die Länder und Gemeinden der Institution an. ${ }^{42}$ So hat nach $\mathbb{S} 24$ Abs. 1 SGB VIII seit dem 1.1.1996 - Übergangsfristen ausgenommen - jedes Kind von der Vollendung des dritten Lebensjahres bis zur Einschulung einen gesetzlichen Anspruch auf den „Besuch einer Tageseinrichtung“. 43 Diese im Hinblick auf die Bezeichnung „Tageseinrichtung“ allgemein gehaltene Bestimmung wird in Landesgesetzen nicht nur wiederholt, sondern spezifiziert. Kindergärten werden als für die Betreuung von Kindern im Alter vom vollendeten dritten Lebensjahr bis zur Einschulung zuständig definiert. ${ }^{44}$ Zusätzlich nor-

41 Statistische Ämter des Bundes und der Länder, Kindertagesbetreuung regional 2010. Ein Vergleich aller 412 Kreise in Deutschland, Wiesbaden 2011, S. 18.

42 Zum Institutionsbegriff und seinen Merkmalen: Hans Merkens, Pädagogische Institutionen. Pädagogisches Handeln im Spannungsfeld von Individualisierung und Organisation, Wiesbaden 2006, S. $31 \mathrm{~m}$. w. N.

43 Das SGB VIII ist als Artikel 1 des Kinder- und Jugendhilfegesetzes (KJHG) vom 26. Juni 1990, BGBl. I S. 1163 normiert worden. Bis zum 31.12.1995 machte $\$ 24$ SGB VIII den Anspruch auf einen Kindergartenplatz von der Maßgabe des jeweiligen Landesrechts abhängig.

44 Vgl. z. B. $\$ 1$ Thüringer Gesetz über die Bildung, Erziehung und Betreuung von Kindern in Tageseinrichtungen und in der Tagespflege als Ausführungsgesetz zum Achten Buch Sozialgesetzbuch - Kinderund Jugendhilfe- (Thüringer Kindertageseinrichtungsgesetz - ThürKitaG -) vom 16.12.2005, GVBl. 2005, S. 365; $\mathbb{~} 1$ Kindertagesstättengesetz Rheinland-Pfalz (KTagStG RP) vom 15.3.1991, GVBl. 1991, S. 79; $\mathbb{1} 1$ Gesetz über die Tageseinrichtungen für Kinder (KiTaG) Niedersachsen in der Fassung vom 7.2.2002, Nds. GVBl. 2002, S. 57; $\$ 1$ Sächsisches Gesetz zur Förderung von Kindern in Tageseinrichtungen (SächsKitaG) vom 15.5.2009, SächsGVBl. 2099, S. 225; $\$ 2$ Gesetz zur Förderung von Kindern in Kindertageseinrichtungen und in der Kindertagespflege (Kindertagesförderungsgesetz KiföG M-V) vom 1.4.2004, GVOBl. M-V 2004, S. 146; \$ 1 Gesetz über die Betreuung und Förderung von Kindern in Kindergärten, anderen Tageseinrichtungen und der Kindertagespflege (KiTaG) BadenWürttemberg vom 19.3.2009, GBl. 2009, S. 161. 
mieren die Landesgesetzgeber in unterschiedlicher Ausführlichkeit die Aufgaben und Ziele der Kindergärten sowie diesbezügliche pädagogische Grundsätze. ${ }^{45}$

Während der Staat genau genommen lediglich Leitentscheidungen zur Funktion und Ausgestaltung der Kindergärten in Recht setzt, führen das gesellschaftliche Engagement und die Akzeptanz dieser Einrichtungen in der Bevölkerung dazu, dass die verbindlich normierten Altersgrenzen und pädagogischen Leitsätze, die im engeren Sinne an die Betreiber von Kindergärten gerichtet sind, faktisch die Ausgestaltung einer Lebensphase bewirken. Die Lebensphase „Kleinkind“ stellt sich so als ein aus dem Zusammenspiel von Staat und Gesellschaft entstehendes Konstrukt dar.

Dessen Ursprünge liegen im 19. Jahrhundert. ${ }^{46}$ Auch wenn diese Zeit emotional als weit zurückliegend empfunden werden mag, Staat und Gesellschaft in der Retrospektive allgemein „eisern“, fast herzlos und antiquiert erscheinen, wirken doch damalige Entscheidungen bis heute fort. Merkmale, wie beispielsweise die Ansiedelung außerhalb des Schulsystems und die Freiwilligkeit des Besuches, haben überdauert und prägen weiterhin die Verortung der vor allem in der historischen Bildungsforschung unter dem Begriff „öffentliche Kleinkindererziehung“ zusammengefassten Einrichtungen in der Gesellschaft. ${ }^{47}$ Dabei wird die Frage nach ihrem Platz in der Gesellschaft regelmäßig diskutiert, wie die immer wieder aufflammenden Kontroversen um eine Kindergartenpflicht und Initiativen wie das Projekt „Schulreifes Kind“ 48 beispielhaft zeigen. In diesen Diskussionen geht es zumeist um eine best-

45 Besonders ausführlich: Präambel und $\mathbb{1} 1$ KiföG M-V; im Übrigen bspw.: $\mathbb{} 6$ ThürKitaG, $\mathbb{} 1 \mathrm{f}$. KTagStG RP, $\mathbb{2}$ f. KiTaG Niedersachsen, $\mathbb{2}$ SächsKitaG, $\mathbb{2}$ KiTaG Baden-Württemberg.

46 Als zeitlicher Rahmen für die hier verfolgte Entwicklung der öffentlichen Kleinkindererziehung bietet sich die Idee des „langen“ 19. Jahrhunderts von 1789 bis 1914 an, zu den verschiedenen Möglichkeiten, das 19. Jahrhundert einzugrenzen: Jürgen Osterhammel, Die Verwandlung der Welt. Eine Geschichte des 19. Jahrhunderts, München 2009, S. 84 ff.

47 Die heute allgemein gebräuchliche Bezeichnung „Kindergarten“ eignet sich für die historische Betrachtung nicht, da bis in das frühe 20. Jahrhundert der „fröbelsche Kindergarten“ mit seiner speziellen Pädagogik Friedrich Fröbels (1782-1852) neben Kleinkinderbewahranstalten und Kleinkinderschulen nur einen Teil der Kinder betreuende Einrichtungen ausmachte. Zur Begrifflichkeit: Günter Erning, Zum Begriff der „öffentlichen Kleinkindererziehung“. Vorüberlegungen zu einer Geschichte der öffentlichen Kleinkindererziehung in Deutschland, in: Sozialpädagogische Blätter 5 (Sept./Okt. 1979), S. 135-142, S. 136. Da sich die Bezeichnung „öffentliche Kleinkindererziehung “ etabliert hat, wird der Begriff auch in dieser Arbeit genutzt. Gleichwohl soll nicht verschwiegen werden, dass es unterschiedliche Ansichten zu den Entwicklungsphasen des Kindes gibt. Die Entwicklungspsychologie unterscheidet beispielsweise je nach dem Stand der biologisch-physiologischen, psychischen und sozialen Entwicklung eines Kindes folgende Entwicklungsabschnitte: Neugeborenes (bis 10. Lebenstag), Säuglingsalter (1. Lebensjahr), Kleinkindalter (2. und 3. Lebensjahr), frühe Kindheit (4.-6. Lebensjahr), mittlere Kindheit (7.-10. Lebensjahr) und späte Kindheit (11.-14. Lebensjahr). Vgl. Artikel „Kind“ in: Brockhaus Enzyklopädie, Band 15, 21. Auflage, Leipzig 2006, S. 5-7, S. 5. Nach dem Pädagogen Herbert Günther erstreckt sich die Kleinkindphase hingegen vom zweiten bis einschließlich fünften Lebensjahr, ders., Sprache hören- Sprache verstehen. Sprachentwicklung und auditive Wahrnehmung, Weinheim 2008, S. 36.

48 Seit 2007 wird mit dem Projekt „Schulreifes Kind“ das Ziel verfolgt, Entwicklungsverzögerungen bei Vorschulkindern frühzeitig zu erkennen und durch gezielte Fördermaßnahmen auszugleichen. Dazu entwickelte das baden-württembergische Ministerium für Kultus, Jugend und Sport ein verzahntes Förderkonzept für Kindergärten und Grundschulen. 
mögliche Vorbereitung auf den Schulbesuch, gerade „[d]ort, wo die sozialen Kompetenzen der Eltern gleich null sind“. 49

Protagonisten der öffentlichen Kleinkindererziehung des 19. Jahrhunderts käme die hier angedeutete Debatte sicher vertraut vor. Ähnelt sie doch der damaligen Situationsbeschreibung der gesellschaftlich relevanten Kräfte. Wie unter anderem diese Wahrnehmung zur Entstehung und Verbreitung der öffentlichen Kleinkindererziehung führte, welche Rolle der Staat dabei spielte und wie die im Zusammenwirken von gesellschaftlichem Engagement, staatlicher Unterstützung und Normierungen geformte Institution „öffentliche Kleinkindererziehung“ die Zeit zwischen dem Säuglings- und dem Schulalter als klar umrissene Lebensphase „Kleinkind“ entstehen ließ, soll am Beispiel Bayerns nachgezeichnet werden.

Die zentrale These des Aufsatzes besagt, dass der bayerische Staat an dieser Entwicklung einen ganz erheblichen Anteil hatte. Er legte einerseits Altersgrenzen fest, erkannte und nutzte aber auch früh die sich durch die öffentliche Kleinkindererziehung ergebende Möglichkeit, die Erziehung der Kinder zu beeinflussen. Bayern steht dabei beispielhaft für eine Entwicklung, wie sie auch in anderen Ländern zu verzeichnen ist.

\section{Die Sozialisation des Kleinkindes und die Behandlung gefährdeter Kinder im frühen 19. Jahrhundert}

Um 1830 entstanden in zahlreichen deutschen Städten und so auch in Bayern Einrichtungen öffentlicher Kleinkindererziehung. ${ }^{50}$ Der Gründung sogenannter Kleinkinderbewahranstalten oder Kleinkinderschulen lagen im Wesentlichen zwei Erkenntnisse der für die Diskussion in der Gesellschaft relevanten adeligen und bürgerlichen Kreise und der öffentlichen Verwaltung zu Grunde. Zum einen wurde seit dem ausgehenden 18. Jahrhundert verstärkt festgestellt, dass Familien der unterbürgerlichen Schichten ihre noch nicht in die Schule oder zur Arbeit gehenden Kinder nur unzureichend beaufsichtigten. Zum anderen war man davon überzeugt, dass mit bürgerlichen Werten erzieherisch auf die unterbürgerlichen Schichten eingewirkt werden muss und sich dazu eine in Anstalten wie z. B. Arbeitshäusern organisierte gruppenweise Betreuung besonders eignet. ${ }^{51}$

49 Heinz Buschkowsky, zitiert nach: Buschkowsky fordert Kindergartenpflicht, in: Der Tagesspiegel vom 3.1.2010, http://www.tagesspiegel.de/berlin/buschkowsky-fordert-kindergartenpflicht/ 1658048.html.

50 Jürgen Reyer, Wenn die Mütter arbeiten gingen ... eine sozialhistorische Studie zur Entstehung der öffentlichen Kleinkindererziehung im 19. Jahrhundert in Deutschland, Köln 1985, S. 19.

51 Friedrich Spaur, Ueber die Pflicht des Staates die Arbeitsamkeit zu befördern, die Betteley abzustellen und die Armen zu versorgen, Salzburg 1802, S. 34, S. 60, S. 354; Angelika Baumann, Armuth ist hier wahrhaft zu Haus ... : Vorindustrieller Pauperismus und Einrichtungen der Armenpflege in Bayern um 1800, München 1984, S. 13; weitere Nachweise bei Michael Doege, Armut in Preußen und Bayern (1770 - 1840), München 1991, S. 362. 
Kleinkinder waren bisher meist nebenbei erzogen worden. Eine Ausnahme bildete insbesondere das städtische Bildungsbürgertum. Dieses griff auf Konzepte der Aufklärungspädagogen und Philanthropen des 18. und frühen 19. Jahrhunderts wie z.B. Pestalozzi zurück. ${ }^{52}$ Die vielen noch nicht arbeitenden Kinder der städtischen Mittelund Unterschichten waren jedoch vielfach sich selbst überlassen. Sie verbrachten viel Zeit ohne Aufsicht zusammen mit älteren Kindern auf der Straße oder in Wohnungen. ${ }^{53}$

Die prekäre Betreuungssituation wurde durch die Professionalisierung des Schulwesens verschärft. Noch zu Beginn des 19. Jahrhunderts traf man Kleinkinder oft in der Volksschule an. ${ }^{54}$ Diese nahm bisher zwei Aufgaben wahr, fungierte als Bildungsund als Bewahranstalt. Die Schulreformen um 1800 normierten dann u. a. ein festes Schuleintrittsalter mit sechs Jahren. ${ }^{55}$ Trotz der Schulreformen blieb die Bewahrfunktion aber zunächst bestehen, weil die Normierungen, insbesondere Schulpflicht, Einschulung und Klassenunterteilung betreffend, nur langsam durchgesetzt wurden. ${ }^{56}$ Die Kleinkinderbetreuung in der Schule war indes nicht zukunftsfähig, da in Schulverordnungen und Lehrplänen kleine Kinder keine Berücksichtigung fanden. Fortschritte in der Professionalisierung des Schulwesens gereichten so letztendlich den Kleinkindern zum Nachteil. Sie verloren mit zunehmender Durchsetzung der Schulreformen einen sicheren Sozialisationsort und vergrößerten zum Teil das Heer aufsichtloser Kinder. Ihr Betteln und tragische Unfälle in Wohnungen wurden durch

52 Darstellung mit Beispielen in: Jürgen Reyer, Wenn die Mütter arbeiten gingen ... eine sozialhistorische Studie zur Entstehung der öffentlichen Kleinkindererziehung im 19. Jahrhundert in Deutschland, Köln 1985, S. 112 ff.; Karl Reinhold Mühlbauer, Zur Lage des Arbeiterkindes im 19. Jahrhundert. Ein sozial- und bildungsgeschichtlicher Beitrag unter besonderer Berücksichtigung der Verhältnisse im Königreich Bayern, Köln 1991, S. 28.

53 Z. B. beschrieb Benjamin Graf von Rumford (1753-1814), Armenpolitiker und Gründer des Militärhauses an der Au in München bettelnde Kinder in: ders., Kleine Schriften politischen, ökonomischen und philosophischen Inhalts, Bd. 1, Weimar 1797, S. 22. S. $87 \mathrm{ff}$.

54 Christian Lange, Bayerische öffentliche Kleinkindererziehung im 19. Jahrhundert. Die Geschichte einer Institution und ihr Recht, in: Stefan Ruppert (Hrsg.), Lebensalter und Recht. Zur Segmentierung des menschlichen Lebenslaufes durch rechtliche Regelungen seit 1750, Frankfurt am Main 2010, S. 3-27, S. $6 \mathrm{ff}$.

55 Verordnung, das Schulwesen (den Schulbesuch) betreffend, vom 23.12.1802, in: Georg Döllinger, Sammlung der im Gebiete der inneren Staats-Verwaltung des Königreichs Bayern bestehenden Verordnungen, 9. Bd., 3. Teil, München 1838, S. 987.

56 Heinrich Stephani (1761-1850), bayerischer Schulreformer, musste noch 1818 erkennen, dass Schüler oft das ganze Jahr über eingeschult wurden, ders., Von der Nothwendigkeit, nur eine jährliche Aufnahmezeit auch für die Volksschulen festzusetzen und für jede Klasse derselben einen hiernach bestimmten Lehrgang anzuordnen, in: Der baierische Schulfreund, 1818, 11 Bd., S. 67-74, S. 65. Aber nicht nur bei der Einschulung vernachlässigte man die Norm. Aus den Schulstandslisten der Provinz Nürnberg des Jahres 1807 ergibt sich beispielsweise für sieben deutsche Schulen in Nürnberg ein durchschnittlicher Anteil vier- bis fünfjähriger Kinder von 10 \%, Auswertung der Daten der Bärrschen, Bauerschen, Düstauischen, Kiefhaberschen, Portischen, Schleizerischen und Schmidischen Schule, Bayerisches Hauptstaatsarchiv, MInn 16, 22093/7. 
Reisebeschreibungen und Zeitungsartikel in den ersten beiden Dekaden des 19. Jahrhunderts einem breiten Publikum bekannt. ${ }^{57}$

So rückten Kinder, die eine Säuglingsversorgung zwar nicht mehr benötigten, aber auch noch nicht „schulreif“" waren, erstmals als Angehörige einer gefährdeten Bevölkerungsgruppe, namentlich der der Kleinkinder, in den Fokus der Gesellschaft. Diese gefährliche Zeit, man kann sie auch als Phase im Lebenslauf der Kinder unterbürgerlicher Herkunft bezeichnen, begann zwar noch individuell, endete aber bereits mit der Einschulung im normativ festgeschrieben Alter von sechs Jahren. Schon hier zeichnet sich die später umfassend definierte Kleinkindphase ab.

Zusammen mit der Beschreibung der Situation kleiner Kinder in dieser Lebensphase diskutierte die lesende Gesellschaft deren Ursachen und Folgen. Sie stellte einen Zusammenhang zwischen fehlender Beaufsichtigung, Bettel und Armut fest und subsumierte dies unter den Begriff „Verwahrlosung “. ${ }^{58}$ Polizei- und ordnungsrechtliche Normierungen beschrieben als gefährlich erkannte Situationen und mahnten die Aufsichtspflicht der Eltern an. ${ }^{59}$ Staat und private Wohltäter beließen es aber nicht bei Normierungen und Appellen. Sie öffneten im öffentlichen Armenwesen und in der Privatwohltätigkeit vorhandene Arbeitsanstalten für kleine Kinder. ${ }^{60}$ Als Teil einer allgemeinen Ausdifferenzierung der Tätigkeit im sozialen Bereich entstanden vereinzelt spezielle Einrichtungen, die auch außerhalb des Schulwesens Kinder zeitweilig oder dauerhaft aus Familien herauslösten und gruppenweise im öffentlichen Raum betreuten. Diese Anstalten erlangten in Bayern allerdings bis in die 1820er Jahre keine überregionale Bedeutung und waren zum Teil sehr kurzlebig. ${ }^{61}$

Neuen Schwung in die Diskussion um „verwahrloste“ Kleinkinder brachten Berichte über erfolgreiche Einrichtungen öffentlicher Kleinkindererziehung vor allem in Eng-

57 Vgl. z. B.: Ueber den Bettel und die häufigen Fallgitter in Baiern, in: Churfürstlich-pfalzbaierisches Regierungs- und Intelligenz-Blatt 1801, S. 344-349, S. 346 f.; Ueber Vernachlässigung der Unterklassen.(Ein Auszug aus dem 4ten Bande „der kleinen Reden an künftige Volksschullehrer) in: Theoretischpractisches Handbuch für deutsche Schullehrer und Erzieher Band 1 (1808) Heft 2, S. 108-129, S. 112; dazu auch: Jürgen Reyer, Wenn die Mütter arbeiten gingen ... eine sozialhistorische Studie zur Entstehung der öffentlichen Kleinkindererziehung im 19. Jahrhundert in Deutschland, Köln 1985, S. 100.

58 Z. B. in: Jäger, Ueber die Schädlichkeit des Viehhütens durch die Kinder, in: Der baierische Schulfreund, Heft 4 (1812), S. 82-90, S. 84.

59 Z. B.: Provinzialverordnung der Königlichen Landes-Direktion München, die nöthige Vorsicht gegen Feuersgefahr betreffend, vom 11.7.1806, in: Königlich-Baierisches Regierungsblatt 1806, S. 275; $\mathbb{} 20$ der Instruktion für die Polizei-Inspektoren in der Haupt- und Residenzstadt München, veröffentlicht mit der Bekanntmachung, die Aufstellung von Polizei=Inspektoren in der Stadt München betreffend, vom 13.11.1812, Königlich-Baierisches Regierungsblatt 1812, S. 1945.

60 Vgl. z. B. die besonderen Maßnahmen Benjamin Graf von Rumfords in dem von ihm gegründeten Münchener Arbeitshaus, ders., Kleine Schriften politischen, ökonomischen und philosophischen Inhalts, Bd. 1 1797, S. $87 \mathrm{ff}$.

61 Zu derartigen Einrichtungen in Augsburg: Susanne F. Eser, Verwaltet und verwahrt Armenpolitik und Arme in Augsburg, Sigmaringen 1996, S. 282; in Regensburg: Karl G. Kick, Von der Armenpflege zur Sozialpolitik, Regensburg 1995, S. 98 f. 
land, die seit ca. 1824 verstärkt veröffentlicht wurden. ${ }^{62}$ Derartige Publikationen endeten häufig mit dem Aufruf, Kleinkinderbewahranstalten oder -schulen auch in deutschen Ländern zu gründen. Als erste bayerische Behörde schloss sich die Königliche Regierung des Rezatkreises diesem Aufruf an. 1828 lobte sie im eigenen Amtsblatt die Einrichtungen öffentlicher Kleinkindererziehung in der Hoffnung, sie mögen eine rasche Verbreitung finden. ${ }^{63}$

\section{Die öffentliche Kleinkindererziehung als schichtspezifisch die Kleinkindphase prägende Institution}

Tatsächlich, aber nicht monokausal auf den Aufruf zurückzuführen, wurden in den folgenden Jahren zahlreiche Anstalten meist von Vereinen gegründet. Zunächst zur Planung der Anstalten und dann in den Verwaltungsausschüssen bzw. Komitees der Kleinkinderschulen und -bewahranstalten trafen sich Lehrer höherer Lehranstalten, Pfarrer, Verwaltungsbeamte, Kaufleute und andere Gewerbetreibende. ${ }^{64}$ Zielgruppe waren Kinder aushäusig arbeitender Eltern, somit solche aus Taglöhner- und Dienstbotenfamilien, Familien von Handwerkergesellen, in den frühindustrialisierten Gebieten Arbeiterfamilien. ${ }^{65}$ Dem ging die allgemein unter den Initiatoren bestehende Erkenntnis voraus, dass es eine „traurige, aber bekannte Wahrheit [sei], daß Hunderte von hoffnungsvollen Kindern schon in den ersten Lebensjahren aus Mangel an

62 Berichte z. B. in der Allgemeinen Schulzeitung zur englischen „infant school“: Allgemeine Schulzeitung (Nr. 22) vom 17.3.1824, S.177-179; Allgemeine Schulzeitung (Nr. 86) vom 27.10.1824, S.745-749, fortgesetzt in der Ausgabe (Nr. 87) vom 30.10.1824, S. 752-756; zur fröbelschen Erziehungsanstalt: Christian Zeh, Die Fröbelsche Erziehungsanstalt zu Keilhau, Allgemeine Schulzeitung (Nr. 85) vom 23.7.1825, S. 673-677.

63 Königliche Regierung des Rezatkreises, Kammer des Innern, die Kleinkinder=Schulen betreffend, in: Königlich Bayerisches Intelligenzblatt für den Rezat=Kreis 1828, S. 1329 f. Bereits im Oktober 1828 lag die Erklärung im Entwurf vor, Staatsarchiv Nürnberg, Reg. v. Mfr. Abg. 1932 Tit.XIII, Nr. 968.

64 Vgl. die Ausschuss- bzw. Vorstandsmitglieder der einzelnen Anstalten aufgeführt als Unterzeichner z. B. in: Das Committee zur Errichtung einer Kleinkinder=Schule in Ansbach, Bitte an ein menschenfreundliches edles Publikum, Königlich-Bayerisches Intelligenzblatt für den Rezatkreis 1832, S. 1163-1164; auch im Bericht des Pfarrrers Rehm an den Nürnberger Magistrat vom 5.7.1833, dem Bericht des Magistrats an die Königliche Regierung des Rezatkreises, Kammer des Innern, zum Rescript vom 21.6.1833, die Kleinkinderschulen betr., beigefügt, Staatsarchiv Nürnberg, Reg. v. Mfr. Abg. 1932 Tit. XIII, Nr. 968.

65 Äußerungen und Berichte der Anstaltgründer in: Der Ausschuss für die Kinderbewahr-Anstalt zu München, Allgemeine Bestimmungen, die Kinder=Bewahranstalt zu München betreffend, München, 31. Oktober 1833, Nr. 1, Druckschrift aus der Staatsbibliothek München. Besonders deutlich wird dies auch im Bericht des Vorstehers der Wöhrder Kleinkinderschule: „Der Hauptzweck der Anstalt ist: den großen Theils verarmten und ihren täglichen Unterhalt außer dem Hause suchen müssenden Eltern die Aufsicht über ihre Kinder abzunehmen, diese von dem so verderblichen Herumsträunen auf den Gassen zu entfernen, sie auf eine nützliche dem kindlichen Alter angemessene Weise zu beschäftigen und schon frühzeitig das Denkvermögen sowohl, als das religiöse Gefühl in ihnen zu wecken“, Bericht des Pfarrrer Loesch an den Nürnberger Magistrat vom 3.7.1833, dem Bericht des Magistrats an die k. Regierung des Rezatkreises, Kammer des Innern, zum Rescript vom 21.6.1833, die Kleinkinderschulen betreffend, beigefügt, Staatsarchiv Nürnberg, Reg. v. Mfr. Abg. 1932 Tit. XIII, Nr. 968. 
der nöthigen Aufsicht und Erziehung einem augenscheinlichen Verderben preisgegeben werden" .66

Die ersten Kleinkinderschulen und -bewahranstalten entstanden nicht im rechtsfreien Raum, sie wurden zumeist als private Erziehungsinstitute von den Distriktschulund Polizeibehörden genehmigt und beaufsichtigt ${ }^{67}$ Eine umfassende Normierung der öffentlichen Kleinkindererziehung gab es gleichwohl noch nicht. Insofern waren deren Gründer für die innere Ausgestaltung der Kleinkinderschulen und -bewahranstalten in dieser Phase besonders wichtig. Dass sie auf nahezu identische Weise die im Wesentlichen gleichen Ziele verfolgten, beruhte auch auf der vorhandenen Literatur zu diesem Thema, die sich einerseits - schon anhand der Bezeichnung „Kleinkinderschule" erkennbar - am Schulwesen orientierte, andererseits aber auch den aus dem Armenwesen bereits bekannten Gedanken, als gefährdet angesehene Kinder aus der Familie herauszulösen und in Einrichtungen gesammelt zu betreuen, aufgegriffen hatte. ${ }^{68}$ Diese „mussten“ nur noch beweisen, dass sie nicht bloß eine vorübergehende Erscheinung waren.

Ebenso war der Institutionalisierung und Synchronisierung der Kleinkindphase das Feld bereitet. Die Zielgruppe der Kleinkinderschulen und -bewahranstalten wurde nicht nur schicht-, sondern auch altersspezifisch umrissen. Während in den Satzungen der Einrichtungen die Abgrenzung „nach unten“ aber noch variabel gestaltet war, endete sie „nach oben hin“ einheitlich mit der Schulpflicht, dem Statuswechsel

66 Der Ausschuss für die Kinderbewahr-Anstalt zu München, Oeffentliche Anzeige, die Errichtung einer Bewahranstalt für kleine Kinder zu München betreffend, München, 31. Oktober 1833, 1. Abschnitt; gleiche Feststellung: Das Committee zur Errichtung einer Kleinkinder=Schule in Ansbach, Bitte an ein menschenfreundliches edles Publikum, Königlich-Bayerisches Intelligenzblatt für den Rezatkreis 1832, S. 1163-1164; ebenso in dem Unterstützungsgesuch des Pfarrers Loesch an die Königliche Regierung des Rezatkreises vom 16.4.1832, Staatsarchiv Nürnberg, Reg. v. Mfr. Abg. 1932 Tit. XIII, Nr. 968 und auch in der Druckschrift „Einige Worte über Kleinkinderschulen“ des freywilligen Vereins, die Errichtung einer Verwahrungsanstalt für kleine Kinder betr., vom 1.5.1831, S. 4, Staatsarchiv Nürnberg, Reg. v. Mfr. Abg. 1932 Tit. XIII, Nr. 968.

67 Grundlage waren seit 1833 die Nr.1-3 der Verordnung, das gemeinsame Wirken der Distrikts=Schulinspectoren und der Distrikts=Polizeibeamten betr., vom 24.7.1833, in: Georg Döllinger, Sammlung der im Gebiete der inneren Staats-Verwaltung des Königreichs Bayern bestehenden Verordnungen, 9. Bd., 3. Teil, München 1838, S. 1071-1074.

68 Samuel Wilderspin, Ueber die frühzeitige Erziehung der Kinder und die englischen Klein-Kinder-Schulen, oder Bemerkungen über die Wichtigkeit, die kleinen Kinder der Armen im Alter von anderthalb bis sieben Jahren zu erziehen, nebst einer Darstellung der Spitalfelder Klein=Kinder=Schule und des daselbst eingeführten Erziehungssystems. Aus dem Englischen nach der dritten Auflage, mit Benützung der neuesten Schriften von W. Wilson, Brown, Mayo u. a., und mit Anmerkungen und Zusätzen versehen von Joseph Wertheimer, 2. Auflage, Wien 1828; Carl John, Die Kleinkinderschule für Kinder von 2 bis 6 Jahren. Vortheile derselben in moralischer und physischer Hinsicht, nebst beigefügtem Lehrplan und Methode, Nordhausen 1830. 
vom Klein- zum Schulkind ${ }^{69}$ Schon die ersten Einrichtungen der öffentlichen Kleinkindererziehung verfestigten so die in den Normierungen zum Schulwesen angedachte, aber zunächst in der Praxis nicht erreichte Trennung der Klein- und Schulkinder.

Zehn Jahre nach ihrem vermehrten Auftreten in Bayern entwarf der bayerische Staat für die Einrichtungen der öffentlichen Kleinkindererziehung mit seinen Allgemeinen Bestimmungen, die Errichtung und Beaufsichtigung der Kleinkinder-Bewahranstalten betreffend von 1839 ein normatives Korsett, welches in seiner Ausführlichkeit in dieser Zeit im deutschsprachigen Raum einmalig war. ${ }^{70}$ Die Normierungen deckten nun im Unterschied zu ersten rudimentären Äußerungen in der Instruction über die Behandlung des Armenwesens vom 24.12.1833 ${ }^{71}$ alle für den Staat relevanten Bereiche der öffentlichen Kleinkindererziehung ab. Sie erstreckten sich von detaillierten pädagogischen Anweisungen bezüglich Ausbildung des Personals und Finanzierung der Einrichtungen bis zum Genehmigungsverfahren und zur ständigen Aufsicht. In bisher kaum erreichtem Umfang wurden pädagogische Überzeugungen für eine Bevölkerungsgruppe - die Kleinkinder - zur Norm erhoben. So wie im Schulrecht Lehrpläne Bildungsinhalte festschrieben, legten die Allgemeinen Bestimmungen die Art der Beschäftigung und das Erziehungsziel fest. ${ }^{72}$ In dieser Pädagogisierung des Rechts trat auch das Bestreben des vormärzlichen Obrigkeitsstaates hervor, die Menschen möglichst früh mit religiös-sittlicher Erziehung zu disziplinieren.

69 Für die Ansbacher Kleinkinderschule: Das Committee zur Errichtung einer Kleinkinder=Schule in Ansbach, Ideen zur Errichtung einer Kleinkinderschule zu Ansbach, Extra=Blatt Nr. 8 zum Rezatkreis=Intelligenzblatt 1832, S. 65-72, $\mathbb{S} \mathbb{S} 1$ u. 40; auch: Schulgesetze der hiesigen Kleinkinderschule nahestehend zur öffentlichen Kenntnis gebracht, vom 25.2.1833, Königlich-bayerisches Intelligenzblatt für den Rezatkreis 1833, S. 321-323;

für die Münchener Kleinkinderbewahranstalt: Altersangaben in: Der Ausschuss für die KinderbewahrAnstalt zu München, Allgemeine Bestimmungen, die Kinder=Bewahranstalt zu München betreffend, München, 31. Oktober 1833, Nr. 1; Bezug zum Erreichen der Schulpflicht in Nr. 6 der Allgemeinen Bestimmungen;

für die Nürnberger Kleinkinderschule: Altersangaben in der Druckschrift „Einige Worte über Kleinkinderschulen“ des freywilligen Vereins, die Errichtung einer Verwahrungsanstalt für kleine Kinder betreffend, vom 1.5.1831, S. 4 u. 13; Bezug zum Erreichen der Schulpflicht in dem Schreiben an die Königliche Regierung des Rezatkreises, Kammer des Innern, vom 14.6.1831,unterthänigste Bitte eines freywilligen Vereins, die Errichtung einer Verwahrungsanstalt für kleine Kinder betreffend, jeweils Staatsarchiv Nürnberg, Reg. v. Mfr. Abg. 1932 Tit. XIII, Nr. 968.

70 Die Bestimmungen wurden unter dem 17.10.1839 im Staatsministerium des Innern entworfen, vom König genehmigt und unter dem 4.11.1839 vom Ministerium des Innern veröffentlicht, Allgemeine Bestimmungen, die Errichtung und Beaufsichtigung der Kleinkinder-Bewahranstalten betreffend, z. B. in: Intelligenz-Blatt von Unterfranken und Aschaffenburg des Königreichs Bayern 1839, 16. besondere Beilage.

71 Instruction über die Behandlung des Armenwesens vom 24.12.1833, in: Georg Döllinger, Sammlung der im Gebiete der inneren Staats-Verwaltung des Königreichs Bayern bestehenden Verordnungen, 12. Bd., München 1838, S. 303.

72 Vgl. den Schulplan vom 8.2.1829, in: Georg Döllinger, Sammlung im Gebiete innern Staats-Verwaltung Bayern bestehenden Verordnungen 9. Bd., 2. Teil, München 1838, S. 589-632; die Schulordnung vom 13.3.1830, die bis zu ihrer Revidierung 1854 mit einigen Änderungen galt. 
Die Aktivitäten in den Bewahranstalten sollten dem Alter der Kinder entsprechen. „Unternehmer sowohl als die Aufsichtsbehörden“ hatten unter anderem darüber zu wachen, dass der , jugendliche Frohsinn durch eine, erst für spätere Lebensjahre geeignete Erziehungsweise nicht verkümmert, der Schule nicht vorgegriffen, und die erst im Heraustreten begriffene Geisteskraft weder übermäßig angestrengt noch unpassend [...] beschäftigt “ werden. ${ }^{73}$ Dementsprechend musste es „eine Hauptaufgabe bleiben, die gesammte Beschäftigungsweise der Kinder zu einem fortwährenden Spiele, $[\ldots]$ zu einer leichten und geregelten, zu einer anregenden, anziehenden und zweckmäßig abwechselnden Unterhaltung zu machen.“74 Die Bezeichnung „Kleinkinderschule" wurde verboten. ${ }^{75}$

Bei den Vorgaben zur kindgerecht anmutenden Erziehung dachte das Staatsministerium auch über die von ihr als „zartes Jugendalter" ${ }^{\text {"76 }}$ bezeichnete kleinkindliche Lebensphase hinaus hochgradig staatswohl- und auf das spätere Erwerbsleben orientiert: Da der „bei weitem [...] größere Theil der in diesen Anstalten aufgenommenen Kinder, armen Eltern angehört" und diese „für einen Stand erzogen werden “ sollten, „welcher vorzugsweise einen gesunden, kräftigen und gewandten Körper, Lust und Liebe zu anstrengender Arbeit und möglichste Beschränkung seiner Bedürfnisse zu seinem künftigen Fortkommen und zu seinem äußern Lebensglücke nöthig hat", so war alles zu vermeiden, „was nachtheilig auf den Gesundheitszustand einwirkt [...], schwächt und verweichlicht". Es durften keine Bedürfnisse geweckt werden, die später „nicht mehr befriedigt werden können“ und daher zur „Unzufriedenheit“ und „Unfrieden“ führen würden. ${ }^{77}$ Erziehung war also in der schichtspezifischen Einrichtung „Kleinkinderbewahranstalt“ nicht allein über Zucht und Zwang, sondern durch ein komplexes Programm auszuüben, welches die erwünschten standestypischen Eigenschaften in den Kindern als eine Art natürliches Verlangen entwickeln sollte.

Die Vorschriften sollten nicht nur gewährleisten, dass sich das in diesem Bereich dominierende privatwohltätige Engagement ganz auf die Erreichung des mit den Kleinkinderbewahranstalten staatlicherseits verbundenen Ziels - der frühzeitigen Verhinderung von „Verwahrlosung“ - konzentriert. Man wollte auch sicherstellen, dass die Einrichtungen auf die im Wesentlichen gleiche Art und Weise ihren Zweck verfolgten. Damit förderten die Normierungen eine uniforme Ausrichtung der bayerischen öffentlichen Kleinkindererziehung, die schon allein deshalb im Großen und Ganzen nicht auf Gegenwehr stieß, da sie mit den Überzeugungen der Einrichtungsträger weitgehend übereinstimmte.

73 Nr. 3 der Allgemeinen Bestimmungen von 1839.

74 Nr. 5 der Allgemeinen Bestimmungen von 1839.

75 Nr. 2 der Allgemeinen Bestimmungen von 1839.

76 Ebenda.

77 Nr. 4 der Allgemeinen Bestimmungen von 1839. 
Mit ihren auf die Kleinkinder abgestimmten Erziehungsrichtlinien trennte das Staatsministerium diese gleichzeitig von den Schulkindern. Passend dazu hatte es schon in Nr. 2 der Normierung mit der Formulierung „für die öffentliche Schule noch nicht reifen Kindern " auf den Entwicklungsgrad und nicht auf das bloße Schuleintrittsalter abgestellt. Die Unterscheidung von Klein- und Schulkindern und damit auch der Übertritt, d. h. der Statuswechsel in die Lebensphase "Schulkind“ erschien somit nicht willkürlich, sondern biologisch bedingt. Im Wissen um die klare Abgrenzung durch das normierte Schuleintrittsalter konnte in den Allgemeinen Bestimmungen auf die Nennung der Altersgrenze verzichtet werden, zumal die bestehenden Kleinkinderbewahranstalten den normierten Statuswechsel in ihre Satzungen und Statuten aufnahmen und nicht in Frage stellten. ${ }^{78}$ Indem aber für die Kleinkinderbewahranstalten auf biologische Gegebenheiten Bezug genommen wurde, stellte sich das Staatsministerium gegen eine Integration der öffentlichen Kleinkindererziehung in das Schulwesen. Kleinkinder waren eben noch nicht schulreif, sollten im Unterschied zu heute nicht gebildet, sondern erzogen werden. Gleichzeitig definierte der Normgeber mit seinen auf den Entwicklungsstand der noch nicht schulfähigen Kinder abgestimmten Erziehungsvorstellungen die Lebensphase „Kleinkind“.

Hoheitliche Regelungen begrenzten diesen Lebensabschnitt nach oben hin nun einerseits durch das konkrete Schuleintrittsalter und andererseits durch die den Kindern in den Allgemeinen Bestimmungen zugesprochenen Fähigkeiten. Für die Abgrenzung nach unten wurde auf die Vorgabe eines bestimmten Alters verzichtet. Die Festlegung einer unteren Altersgrenze hätte für den Staat auch kaum einen besonderen Nutzen gehabt. Er griff grundsätzlich, d. h. ohne dass in der Person des Kindes oder in dessen Umfeld besondere Gründe vorlagen, erst mit dem Schulwesen auf die Kinder zu. Eine starre Unterteilung des Lebensabschnittes vor der Einschulung war daher nicht erforderlich. So konnte er die in den Satzungen und Statuten der Kleinkinderbewahranstalten normierten Altersgrenzen problemlos akzeptieren. Diese orientierten sich nicht nur an den örtlichen Gegebenheiten, sondern vor allem an der Entwicklung der Kinder. Die Pflege von Säuglingen vermochten die Einrichtungen regelmäßig nicht zu leisten. Schon aus diesem Grunde war der Betreuung in den Anstalten eine natürliche Grenze gesetzt, die in den Anstaltssatzungen zwischen dem

78 Z. B. Satzung des Vereins Kinderbewahranstalt in Würzburg vom 16.1.1837, $\mathbb{S} 1,17$, Bayerisches Hauptstaatsarchiv, M Inn 52955 Frauenverein bzw. Kleinkinderbewahranstalt in Würzburg, 1837-1863; Allgemeine Statuten für die Kleinkinder-Bewahrschule zu Schwabach vom 25.3.1837, II.; Statuten für die Kleinkinderbewahrschule zu Schwabach vom 22.5.1837, A.III. ff., B.I. beide: Staatsarchiv Nürnberg, Reg. v. Mfr. Abg. 1932 Tit. XIII, Nr. 968; Plan resp. Statuten etc. Neuendettelsau vom 18.4.1838, $\mathbb{S} \mathbb{S} 1$, 4, Staatsarchiv Nürnberg, Reg. v. Mfr. Abg. 1932 Tit. XIII, Nr. 968; Erster öffentlicher Jahresbericht über die Kleinkinder=Bewahranstalt des Frauen-Vereins zu Landau in der Pfalz 1838, S. 3 f, 6, dort auch noch einmal ausführlich zur Situation der Kinder und den ihnen drohenden Gefahren. 
zweiten und dem vierten Lebensjahr verortet wurde. ${ }^{79}$ Letztendlich bildete sich so auch ohne staatliche Normierung die untere Begrenzung der Kleinkindphase heraus.

Schon zu dieser Zeit stellte sich also die bewusst wahrnehmbare Kleinkindphase als ein aus dem Zusammenspiel von Staat und Gesellschaft entstehendes Konstrukt dar. Der Schwerpunkt der Verrechtlichung dieser Lebensphase lag dabei in deren oberer Begrenzung und pädagogischen Ausgestaltung. ${ }^{80} \mathrm{Im}$ Unterschied zur heutigen Situation war sie allerdings noch schichtspezifisch konzipiert und damit längst noch nicht Bestandteil des Lebensverlaufes nahezu aller Kinder.

Mit ihrer schichtspezifischen Ausrichtung hatte die öffentliche Kleinkindererziehung keine reelle Chance auf die Integration in das Schulsystem. Zwar unterstützten die jeweiligen Staatsregierungen und das Königshaus zahlreiche Einrichtungen. ${ }^{81}$ Diese Unterstützung erfolgte aber freiwillig und bezüglich des finanziellen Volumens variabel. Auch aus fiskalischen Erwägungen wollte der Staat sicherlich nicht als Anstaltsträger auftreten, die öffentliche Kleinkindererziehung nicht wie das Schulwesen zur Staatsanstalt erklären.

\section{Die Entwicklung der Anstalten zur schichtübergreifenden Einrichtung}

1839 waren von staatlicher Seite die wesentlichen Vorschriften zur Regulierung und Indienstnahme der Einrichtungen öffentlicher Kleinkindererziehung und ihrer Träger erlassen. Zwei auf thematisch begrenzte Nachbesserungen abzielende Verordnungen von 1846 und 1847 hatten nur noch ergänzenden Charakter. ${ }^{82} \mathrm{Ob}$ es zu einer allgemeinen Prägung der Kleinkindphase durch die Einrichtungen öffentlicher Kleinkindererziehung kommen würde, war nur noch eine Frage der Ausbreitung der

79 Ebenda.

80 Zum Begriff der Verrechtlichung: Rüdiger Voigt, Verrechtlichung in Staat und Gesellschaft, in: ders. (Hrsg.), Verrechtlichung. Analysen zu Funktion und Wirkung von Parlamentarisierung, Bürokratisierung und Justizialisierung sozialer, politischer und ökonomischer Prozesse, Königstein/Ts. 1980, S. 15-37.

81 Als Protektorin des Trägervereins der Würzburger Kleinkinderbewahranstalt spendete die verwitwete Frau König Maximilians I. Joseph, Königin Carolina, 1838 beispielsweise 100 Gulden. Die regierende Königin Therese gab die gleiche Summe, Zweiter Jahres=Bericht über die zu Würzburg bestehende Kleinkinder-Bewahranstalt, vom 1. März 1837 bis 1. März 1838, Würzburg1838, S. 6; die Königin engagierte sich u. a. auch als Schutzherrin der Augsburger Kleinkinderbewahranstalten, Johann Georg Wirth, Kleinkinderbewahranstalten. Geschichte derselben überhaupt, in: Quartalschrift für praktisches Schulwesen: mit besonderer Rücksicht auf das Königreich Bayern, Jahrgang 1 (1837), Heft 1, S. 108-114, fortgeführt als: Kleinkinderbewahranstalten der Kreishauptstadt Augsburg, in: Quartalschrift für praktisches Schulwesen: mit besonderer Rücksicht auf das Königreich Bayern, Jahrgang 1 (1837), Heft 1, S. 114-120, fortgeführt Heft 2, S. 234-239, hier Heft 2 S. 234.

82 Ministerial-Verordnung (des Innern), die Beschäftigungen in den Kleinkinder-Bewahranstalten betreffend, vom 12.6.1846, in: Friedrich von Strauß, Fortgesetzte Sammlung der im Gebiet der inneren Staatsverwaltung des Königreiches Bayern bestehenden Verordnungen von 1835 bis 1852, Vierter Band der neuen Folge Als Fortsetzung der Döllinger'schen Sammlung XXIV. Band, München 1853, S. 593; Ministerial-Verordnung (des Innern für Kirchen- und Schulangelegenheiten), die religiösen Uebungen an den Kleinkinderbewahranstalten betreffend, vom 23.8.1847, in: Bayerisches Hauptstaatsarchiv, MInn 16, 52828, Nr. 6876. 
Kleinkinderbewahranstalten und deren Erweiterung auf die anderen Bevölkerungsschichten.

Im weiteren Verlauf geschah genau dies. Mehr und mehr Anstalten wurden gegründet, zum Ende des 19. Jahrhunderts auch immer öfter schichtübergreifende Kindergärten nach der Pädagogik Friedrich Fröbels. ${ }^{83}$

In Bayern berücksichtigte der Normgeber diese Entwicklung, indem er 1910 in der Neufassung der Allgemeinen Bestimmungen die schichtspezifischen Formulierungen weitgehend strich. Kleinkinderbewahranstalten und Kindergärten hatten nun allgemein „Kindern vor Eintritt in die Volksschule während des Tages Aufenthalt, Pflege und Erziehung zu gewähren “ ${ }^{84}$ Regelungen zum Aufnahmealter grenzten die Kleinkindphase jetzt auch zu den Säuglingen in den seit den 1850er Jahren aufkommenden Kinderkrippen ab. ${ }^{85}$ Nur Kinder, die „einer besonderen Pflege nicht mehr bedürfen“, sollten aufgenommen werden. Hierfür wurde ein Alter von 3 Jahren angesetzt, Abweichungen im Einzelfall aber zugelassen. ${ }^{86}$ Somit war die Kleinkindphase nun vollständig rechtlich umrissen.

Die Vorgaben zur Erziehung in den Anstalten hatten sich in den Augen des Staatsministeriums bewährt; deren früher festgelegter Umfang blieb im Wesentlichen unverändert.

\section{Fazit}

Die Besuchszahlen der Einrichtungen öffentlicher Kleinkindererziehung waren zu Beginn des 20. Jahrhunderts noch weit von ihrem jetzigen Stand entfernt. Auch darf der Beitrag der Pädagogen des 20. Jahrhunderts für das Wirken der heutigen Kindergärten nicht unterschätzt werden.

Trotz allem ist die bis heute in ihren Grenzen beständige Kleinkindphase hauptsächlich ein Ergebnis der Entwicklung im 19. Jahrhundert. Recht hatte hieran einen ganz erheblichen Anteil. Es legte nicht nur die untere und obere Begrenzung des Bausteins

83 Zu Friedrich Fröbel (1782-1852) und seiner Pädagogik: Helmut Heiland/Michael Gebel/Karl Neumann, Perspektive der Fröbelforschung, Würzburg 2006, dort auch eine neuere Bibliographie Friedrich Fröbel. Für das Jahr 1871/72 zählten die bayerischen Statistiker insgesamt 27 Kindergärten im Land, für 1884/85 bereits 75 Einrichtungen. Den Kindergärten standen 1884/85 293 Kleinkinderbewahranstalten gegenüber, Königlich statistisches Bureau, Statistik des Unterrichts und der Erziehung im Königreiche Bayern für die Jahre 1869/70,1870/71 und 1871/72. Zweiter Teil. Die Lehrerbildung, der Elementarunterricht und die Erziehungsanstalten, Ackermann, München 1875, S. 394-427.

84 Nr. 1 der Allgemeinen Bestimmungen über Einrichtung und Betrieb von Kinderbewahranstalten vom 17.12.1910, Ministerialblatt für Kirchen- und Schul-Angelegenheiten im Königreich Bayern 1910, S. 727-737.

85 Z. B. in München und Nürnberg wurden in dieser Zeit Kinderkrippen errichtet, vgl.: Karl August Widmer (Hrsg.), Erster Bericht über die Krippe oder Säuglingsbewahranstalt in München, München 1855; zum Zweck der Krippen ebenfalls: Zweiter Jahresbericht über die Wirksamkeit des lutherischen Hilfsvereins in Nürnberg für weibliche Diakonie in Bayern die Pflege-Anstalt für arme Mädchen und die Krippe für kleine Kinder betreffend, Nürnberg 1858, S. 7.

86 Hierzu insgesamt Nr. 9 der Bestimmungen von 1910. 
im Lebenslauf des Menschen fest, sondern füllte diesen auch mit einem in den Einrichtungen der öffentlichen Kleinkindererziehung umzusetzenden pädagogischen Inhalt.

Gerade der Blick auf die sich im Laufe der Zeit wandelnden pädagogischen Anforderungen ${ }^{87}$ verdeutlicht die Stärke der Lebensphase „Kleinkind“ im Besonderen, aber auch allgemein die Bedeutung der Lebensphasen und Altersgrenzen: Lebensphasen und Altersgrenzen sind besonders dauerhaft, weil sie sich mit unterschiedlichen Begründungen rechtfertigen und mit wechselnden Inhalten füllen lassen.

\section{Hochaltrigkeit im Recht (Kathrin Brunozzi)}

\section{Einleitung}

Die alternde Gesellschaft hat Potentiale, vor allem ältere Menschen werden unterschätzt. Diese Befunde der Alterswissenschaften, vorgetragen als Appell und Feststellung, finden sich immer häufiger. ${ }^{88}$ Seit 2006 versucht etwa ein Projekt in Schleswig-Holstein, dem Rechnung zu tragen: Mit dem Konzept „seniorTrainerin, Erfahrungs-Wissen für Initiativen (EFI)“ sollen ältere Menschen auf kommunaler Ebene „aktiv Verantwortung “ übernehmen. ${ }^{89}$ Die gleiche Person, die mit Mitte Sechzig generationsübergreifende Projekte plant und leitet, könnte sich Mitte Siebzig für den Umzug ins Betreute Wohnen oder in ein Mehrgenerationenhaus entscheiden. Bei Bedarf springt sie für die Versorgung der Enkelkinder ein, entleert während des (Klein-)Familienurlaubs den Briefkasten und gießt die Blumen. Fünfzehn Jahre später hat sich der Gesundheitszustand dieses mobilen Rentners und gleichzeitig unabhängigen, alten Menschen möglicherweise stark verschlechtert. Dies könnte ihn dazu bewegen, den Lebensabend schließlich in einem Pflegeheim zu verbringen. Und das Fazit müsste lauten: Einen Lebenslauf im Alter, in dem sich eine dritte und eine vierte Lebensphase $^{90}$ unterscheiden, gibt es in der Praxis längst.

Aufgrund des demographischen Wandels rücken die mit den Phasen des Alterns verbundenen Fragen zunehmend in den Blickpunkt der Öffentlichkeit. Aus juristischer Perspektive ist dabei interessant, ob ein Lebenslauf, wie der geschilderte, ebenso im Recht erkennbar ist bzw. erkennbar geworden ist. Von dem in der Einleitung dargestellten „Recht des Lebenslaufs“ her betrachtet, stellt sich die Frage, ob und inwiefern auch das Alter rechtlich binnenstrukturiert und ausgestaltet worden ist.

87 Vgl. hierzu die Bildungs-, Erziehungs- und Betreuungsaufgaben in den aktuellen Kindertagesstättengesetzen oder die Liste der Publikationen zu diesem Thema allein schon des VS Verlages für Sozialwissenschaften.

88 Siehe etwa Ursula Staudinger, Zukunft des Alter(n)s, in: Diether Döring/Eduard M. J. Kroker (Hrsg.), Alter und Gesellschaft, Frankfurt am Main 2009, S. 143 ff.; Axel Börsch-Supan, Ökonomische Auswirkungen des demografischen Wandels, in: APuZ 2011, 19-26.

89 http://www.isab-institut.de/front_content.php?idart=422 (12.7.2011).

$90 \mathrm{Zu}$ den Lebensphasen siehe die Einleitung von Stefan Ruppert. 
Überlegungen zu einem allgemeinen „Recht der älteren Menschen “91 sind bereits seit mehr als einem Jahrzehnt Gegenstand rechtswissenschaftlicher Betrachtungen. Einem regelmäßigen Zeitungsleser fallen in dem Zusammenhang möglicherweise die Debatten zu Patientenverfügungen, künstlicher Ernährung oder Sterbehilfe ein. ${ }^{92}$ Neben ethischen geht es in diesen juristisch oft um straf- und verfassungsrechtliche Aspekte. Rechtliche Probleme, die insbesondere alte Menschen tangieren und damit auf ihre Weise „altenspezifisch“ sind, beginnen aber bereits vor dem Übergang vom Lebensende zum Sterben. Sie zeigen sich in juristischen Betrachtungen, die Titel wie

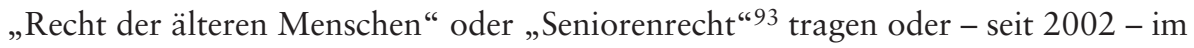
Vierten Altenbericht der Bundesregierung. ${ }^{94}$ Diese Publikationen thematisieren konkret sozialversicherungs-, heim- oder betreuungsrechtliche Fragen, die besonders Menschen im hohen und höchsten Lebensalter berühren.

Dabei ist schwer bestimmbar, wann ein Übergang ins hohe oder höchste Lebensalter stattfindet. Faktisch vollziehen sich der Alternsverlauf und die Übergänge von einem „jungen“ zu einem „alten“ Alter" anhand des Grades der Eigenständigkeit. Eine strikt kalendarische Abgrenzung verschiedener „Altersphasen“ voneinander scheint demgegenüber unmöglich: Gilt man im Alter noch „jung “ mit 66 Jahren, wenn aus der Perspektive einiger womöglich gerade die Zeit der „Freiheit“ anfängt?9 ${ }^{96}$ Ist man „hochaltrig“ bereits, wenn ein nachbarschaftlicher Helfer den täglichen Einkauf übernimmt oder erst, wenn eine Versorgung mit „Pflegestufe 3 “97 unvermeidbar wird? Und in welchem Lebensjahr mögen solche Stadien erreicht sein? Diese Fragen können kaum generell beantwortet werden. ${ }^{98}$ Aller Individualität des Alterns zum Trotz werden Altersphasen dennoch regelmäßig auch mithilfe kalendarischer Maß-

91 Gerhard Igl/Thomas Klie (Hrsg.), Das Recht der älteren Menschen, Baden-Baden 2007.

92 Tatsächlich kommen zwei Drittel der Ernährungsschläuche bei hochbetagten Menschen in Altenheimen und Krankenhäusern zum Einsatz. Annähernd die Hälfte der Betroffenen ist altersverwirrt oder bereits hochgradig dement, Martin Spiewak, Leben am Schlauch, in: DIE ZEIT vom 28.5.2009, Nr. 23.

93 Ronald Richter/Bettina Schmidt/Michael Klatt/Gudrun Doering-Striening (Hrsg.), Seniorenrecht in der anwaltlichen Praxis, Baden-Baden 2006.

94 Der Vierte Altenbericht zur Lage der älteren Generation in der Bundesrepublik Deutschland: Risiken, Lebensqualität und Versorgung Hochaltriger - unter besonderer Berücksichtigung demenzieller Erkrankungen enthält auf S. $319 \mathrm{ff}$. einen eigenen Abschnitt „Rechtliche Rahmenbedingungen und Notwendigkeiten ihrer Fortentwicklung, http://www.bmfsfj.de/bmfsfj/generator/BMFSFJ/Service/Publikationen/publikationen, did=5362.html (25.1.2012).

95 Die Begriffe der „jungen“ und der „alten“ Alten gehen auf Bernice L. Neugarten zurück. Einen Überblick über die Entwicklungen der Gerontologie geben Hans-Werner Wahl/Vera Heyl, Gerontologie. Einführung und Geschichte, Stuttgart 2004.

96 Wie das Alter empfunden wird, hängt nach einer Studie von Tamayo Korte davon ab, welche Erwartungen bezüglich der Einschränkung der Autonomie erwartet werden, Tamayo Korte, Altern - ein Risiko für die Autonomie?, in: FRP 2004, 643-648.

97 Pflegestufe III (Schwerstpflegebedürftige) ist definiert in $\ 15$ Abs. 1 Nr. 3 SGB XI.

98 Diese Fragen stellt auch der Sechste Bericht zur Lage der älteren Generation in der Bundesrepublik Deutschland - Altersbilder in der Gesellschaft, BT-Drs. 17/3815, S. 19. 
stäbe gebildet und der „Beginn“ eines Vierten Alters bei 75 bis 85 Jahren angesiedelt. ${ }^{99}$ Es stellt eine Besonderheit des Rechts dar, dass der Mensch einerseits sowohl zivil- als auch strafrechtlich über Altersstufen in rechtliche Verantwortung hineinwächst. ${ }^{100}$ Andererseits „verliert“ er die einmal gewonnene Verantwortung aber nicht in gleichem Maße stufenweise. ${ }^{101}$ Während der Gesetzgeber Kindern, Jugendlichen und jungen Erwachsenen überwiegend mithilfe von Altersgrenzen Rechte und Pflichten ab- und zuerkennt, ${ }^{102}$ wird man in Gesetzbüchern Altersgrenzen jenseits der 65 nur selten finden. ${ }^{103}$

Trotz dieser Feststellung liegt dem Aufsatz die folgende These zugrunde: Obwohl keine eindeutigen kalendarischen Abgrenzungen zwischen dem „Dritten“ und „Vierten Alter" existieren, haben verschiedene Altersbilder und -wahrnehmungen über die letzten fünf Jahrzehnte Normen hervorgebracht, in denen sich Lebensphasen im Alter spiegeln und die praktisch bestimmte Altersphasen adressieren. Nachdem sich Hochbetagtheit klar abgrenzbaren Altersstufen entzieht, gibt es für Hochaltrige dabei nicht den „einen“ Lebenslauf im Recht. ${ }^{104}$ Vielmehr werden verschiedene Lebensverläufe normiert oder von gesetzlichen Regelungen eben gerade nicht erfasst. ${ }^{105}$

Diese These soll im Folgenden anhand von zwei Rechtsgebieten nachvollzogen werden. Im öffentlichen Recht stellt die Entwicklung des Heimrechts ein Beispiel dar. Zivilrechtliche Aspekte des Alterns zeigen das frühere Recht der „Gebrechlichkeitspflegschaft" und sein in den Neunzigerjahren entstandener Nachfolger, das Betreu-

99 Z.B. Paul B. Baltes, Die Freiheit der Ältesten. Entscheidungen am Lebensende, in: Frankfurter Allgemeine Zeitung (28.10.2005), S. 37; Gerd Göckenjan/Hans-Joachim von Kondratowitz, Altern Kampf um Deutungen und um Lebensformen, in: Gerd Göckenjan/Hans-Joachim von Kondratowitz (Hrsg.), Alter und Alltag, Frankfurt am Main 1988, S.13; Vierter Altenbericht, S. 45 http:// www.bmfsfj.de/bmfsfj/generator/BMFSFJ/Service/Publikationen/publikationen, did=5362.html (25.1.2012).

100 Aus rechtshistorischer Sicht werden die Lebensphasen in jungen Jahren vor allem beleuchtet in den Arbeiten von Tanja Mill, Zur Erziehung verurteilt, Frankfurt am Main 2010 und Thilo Engel, Elterliche Gewalt unter staatlicher Aufsicht in Frankreich und Deutschland (1870-1924), Frankfurt am Main 2011.

101 In Bezug auf den Führerschein wird die Altersgrenze seit mehreren Jahrzehnten diskutiert, siehe etwa „Ältere Fahrer, sichere Fahrer? in: DIE ZEIT vom 17.1.1986 oder: Wann sollen Senioren den Führerschein abgeben?, in: Hannoversche Allgemeine vom 27.11.2010, http:/www.haz.de/Ratgeber/ Auto-Verkehr/Fahrschule-der-HAZ/Wann-sollen-Senioren-den-Fuehrerschein-abgeben (17.2.2011).

102 Beispiele: $\mathbb{S} \mathbb{S} 107,828$ BGB, in $\mathbb{} 19$ StGB sowie im KJHG. Zu aktuellen Altersgrenzen im Recht Gerhard Igl, Zur Problematik der Altersgrenzen aus juristischer Perspektive, in: Zeitschrift für Gerontologie und Geriatrie, Suppl. I 2000, S. 58-70.

103 Ein Beispiel bildet $\$ 9$ Abs. 3 a) Verschollenheitsgesetz.

104 Die Vielfalt des Alterns und seiner Wahrnehmung verdeutlicht u.a. der Sechste Altenbericht, BT-Drs. 17/3815, der sich mit Altersbildern, also Vorstellungen vom Alter, in unterschiedlichen Kontexten beschäftigt.

105 Die folgenden Ausführungen fassen Ergebnisse meiner Dissertation „Das Vierte Alter im Recht. Heimrecht und Betreuungsrecht in der Bundesrepublik" zusammen, im Erscheinen. 
ungsrecht. ${ }^{106}$ An diesen Rechtsgebieten lassen sich drei Beobachtungen aufzeigen, die die Normierung der Alternsphase und deren rechtliche „Binnenstrukturierung“ widerspiegeln: Erstens erfahren Heim- und Betreuungsverhältnisse seit den Sechzigerjahren eine umfassende Verrechtlichung. ${ }^{107}$ Zweitens betraf diese von den Alternsphasen vor allem das hohe Alter. Drittens fand die Entwicklung zum großen Teil statt, ohne dass Juristen die treibenden Kräfte eines Themenfeldes Alter und Recht gewesen wären.

\section{Verrechtlichung}

Das Leben in einer Alteneinrichtung oder unter Bestellung eines Gebrechlichkeitspflegers wurde im 20. Jahrhundert vom Gesetzgeber reguliert und von der Rechtsprechung ausgestaltet.

Auf Initiative der Länder schufen Bundestagsabgeordnete Ende der Sechzigerjahre eine gewerberechtliche Grundlage für die behördliche Kontrolle von (Alten-)einrichtungen. ${ }^{108}$ Da sich die Vorschrift auf privatgewerbliche Betriebe beschränkte, schloss sie nur einen geringen Teil von Heimen ein. ${ }^{109}$ An diese konnten die Länder damit jedoch erste bauliche und personelle Anforderungen stellen. ${ }^{110}$ Der Heimgesetzgebungsprozess setzte Anfang der Siebzigerjahre ein. Nicht nur mussten sich nun auch freigemeinnützige Heime einer behördlichen Aufsicht unterwerfen. Vielmehr trat das Bild des hilflosen, schutzbedürftigen alten Menschen in den Bundestagsdebatten in den Hinter- und der Bewohner mit eigenen Interessen und Bedürfnissen in den Vordergrund. ${ }^{111}$ Der in der Praxis einzelner Heime bereits erprobte Heimbeirat ${ }^{112}$ wurde für alle Einrichtungen obligatorisch. Damit schuf der Gesetzgeber ein Bewohnergremium, das der Heimleitung gegenüberstehen sollte und griff erstmals unmittelbar in

106 Beide Rechtsgebiete finden sich in Leitfaden zu seniorenrechtlichen Fragen, etwa bei Ronald Richter u.a. (Hrsg.), Seniorenrecht in der anwaltlichen Praxis, Baden-Baden 2006; Gerhard Igl/Thomas Klie (Hrsg.), Das Recht der älteren Menschen, Baden-Baden 2007.

107 Dieser Begriff soll zwei Phänomene umschreiben: Rechtliche Regelungen werden auf neue Lebenssachverhalte ausgedehnt, und bereits existierende Normen werden detaillierter und spezialisierter, u.a. Rüdiger Voigt, Verrechtlichung in Staat und Gesellschaft, in: Rüdiger Voigt (Hrsg.), Verrechtlichung. Analysen zu Funktion und Wirkung von Parlamentarisierung, Bürokratisierung und Justizialisierung sozialer, politischer und ökonomischer Prozesse, Königstein/Ts. 1980, S. 15-37, S. 16.

$108 \$ 38$ GewO im Siebten Gesetz zur Änderung der Gewerbeordnung, BGBl. I 1967, S. 933.

109 Nach Zahlen aus dem Jahr 1969 handelte es sich nur bei $9 \%$ aller Einrichtungen um gewerbliche Einrichtungen. Die meisten Altenheime waren in öffentlicher Hand oder wurden von den Wohlfahrtsverbänden betrieben; Zahlen aus dem Bericht der Bundesregierung, BT-Drs. 5/4122 vom 21.4.1969, S. 2.

110 Dazu gehörten Mindestanforderungen an Räume und Personal, jedoch auch Vorschriften für Buchführung oder Kontrollbesuche. Einen Überblick über die Berliner VO gibt Maximilian Zeise, Mindestanforderungen und Überwachungsmaßnahmen gegenüber gewerblichen Alten- und Pflegeeinrichtungen, in: Soziale Arbeit 1967, S. 528-536.

111 Der Umbruch zeigt sich vor allem in einem Vergleich zwischen den Bundesrats- und den Bundestagsdebatten, so etwa im Kurzprotokoll der Sitzung des JFGA am 21.3.1973, Parlamentsarchiv (PA) VII/176 A 2 Dok. 36.

112 Erfahrungsberichte, in: Blätter der Wohlfahrtspflege 1974, S. $317 \mathrm{ff.}$ 
das Verhältnis zwischen Leiter und Bewohner der Einrichtungen ein. Teile ihres Alltags sollten Bewohner nun mitgestalten können. In den folgenden 15 Jahren wurden Selbständigkeit und Selbstbestimmung der Bewohner wieder weniger diskutiert, stattdessen nahmen finanzielle Fragen zur Pflege einen immer breiteren Raum ein. ${ }^{113}$ Pflege- und sozialleistungsrechtliche Probleme sollten jedoch nicht mithilfe des Heimrechts gelöst werden. Dieses behielt einen ordnungsrechtlichen Charakter. Das erste Heimänderungsgesetz zielte daher darauf ab, den Bewohner als Vertragspartner des meist überlegenen Heimträgers zu stärken. ${ }^{114}$ Das zweite Heimänderungsgesetz erstreckte seine Geltung 1997 auf die Kurzzeitpflege. ${ }^{115}$ Auch Einrichtungen, die kranke oder alte Menschen vorübergehend aufnahmen, mussten nun heimrechtliche Vorgaben beachten. Das dritte Heimänderungsgesetz bemühte sich 2001 schließlich angesichts der immer vielfältigeren Wohnformen, den Anwendungsbereich genauer abzugrenzen. ${ }^{116}$ So sollten vor allem Menschen, die sich für Betreutes Wohnen entschieden, außerhalb der Vorgaben des Heimgesetzes leben können.

Während Heimrecht von Länderregierungen und Abgeordneten vorangetrieben wurde, begann im Betreuungsrecht die Rechtsprechung mithilfe verfahrensrechtlicher Vorgaben das Verhältnis zwischen Pfleger und „Gebrechlichkeitspflegling“ richterlicher Kontrolle zu erschließen. ${ }^{117}$ Ein bundesverfassungsgerichtlicher Beschluss von $1960^{118}$ und die sich anschließende Rechtsprechung des Bundesgerichtshofs stärkte die Rechtstellung des Pflegebefohlenen. ${ }^{119}$ Der Pfleger oder Vormund wurde bei seinen Aufgaben vermehrt beaufsichtigt. Eine Ausdehnung verfahrensrechtlicher Grundsätze berechtigte den Betroffenen, in seinem eigenen gerichtlichen Verfahren angehört zu werden. Ein (fach-)ärztliches Gutachten sollte die Grundlage für den

113 Phasen der Entwicklung des Pflegeversicherungsgesetzes bei Gerhard Igl, Leistungen bei Pflegebedürftigkeit, München 1992.

$114 \int \mathbb{S} 4 \mathrm{a}$ bis d zum Heimvertrag in: BGBl. I 1990, S. 758. Gerhard Igl bezeichnet den Heimvertrag in der Anhörung vor dem Bundestag als „Herrschaftsinstrument der Heimträger“, Wortprot. des JFFGA am 15.11.1989, PA XI/224 A Dok. 21, S. 18.

115 BGBl I. 1997, 158; Klaus Füßer, Regulierung und Deregulierung im Heimrecht: Das Zweite Gesetz zur Änderung des Heimgesetzes vom 3. 2. 1997, in: NJW 1997, S. 1957.

116 Das Heimrecht wurde im Rahmen der Föderalismusreform 2006 in die Gesetzgebungszuständigkeit der Länder gegeben. Zu den bereits entstandenen Landesgesetzen Julia Burmeister/Katharina Dinter, Die Heimgesetzgebung der Bundesländer - Ein Rechtsvergleich, NVwZ 2009, 628-636. Für einen Überblick über die Landesgesetze: http://www.biva.de/index.php?id=639 (17.2.2011). Am 1.10.2009 trat das Wohn- und Betreuungsvertragsgesetz in Kraft (BGBl. I 2009, S. 2319), das die durch den Bund zu regelnde Materie des Heimvertrags betrifft.

117 Die Gebrechlichkeitspflegschaft war ursprünglich geregelt in \$1910 BGB. Die gesetzlichen Vorschriften entsprachen bis zur Schaffung des Betreuungsgesetzes weitgehend den seit 1900 geltenden Normen. Der Bundesgerichtshof löste sich erst in den Sechzigerjahren von der Rechtsprechung des Reichsgerichts zu verschiedenen pflegschaftsrechtlichen Grundfragen.

118 Beschluss des BVerfG vom 10.2.1960, in: NJW 1960, S. 811 ff; BVerfGE 10, 320.

119 BGH Beschluss vom 22.3.1961, in: BGHZ 35, 1, 4. Leitsatz: „Gegen eine Verfügung, durch die eine Gebrechlichkeitspflegschaft über einen Volljährigen angeordnet worden ist, kann der Betroffene auch im Falle seiner Geschäftsunfähigkeit wirksam Beschwerde einlegen.". 
Eingriff in die rechtliche Selbständigkeit bilden. In den unteren Instanzen blieb die Umsetzung der höchstrichterlichen Vorgaben in den Folgejahren problembehaftet. In den zivilrechtlichen Normen schlug sich die Entwicklung im Betreuungsgesetz von 1990 nieder. Dieses wurde zwar seit seinem Inkrafttreten mehrfach modifiziert. Das Konzept einer Betreuung, das vorschreibt, Wünsche des Betreuten zu berücksichtigen, und bestimmte Verfahrensgarantien gibt, ist aber unverändert geblieben. ${ }^{120}$

\section{Verrechtlichung des „Vierten Lebensalters“}

Die in der Entwicklung von Heim- und Betreuungsrecht durch Gesetzgebung und Rechtsprechung steckende Verrechtlichung betraf vor allem die Lebensphase des hohen Alters.

Anders als das Pflegschafts- und Betreuungsrecht, war das Heimrecht von Beginn an auf alte und hochaltrige Menschen ausgerichtet. Kommunale Verwaltungsbeamte und Wissenschaftler wiesen schon in den frühen Sechzigerjahren darauf hin, dass Heimbewohner stets zu den Älteren der alten Menschen gehörten. ${ }^{121} \mathrm{Im}$ Vorfeld zur ersten gesetzlichen Regelung standen entsprechend allein die Ausbeutung und die schlechte Versorgung hilfloser alter Menschen zur Debatte. ${ }^{122}$ Bereits im Rahmen der 1965 einsetzenden Gewerbeordnungsänderung wurde befunden, dass sich die Betreuung nach dem Grad der Hilfsbedürftigkeit richte. ${ }^{123}$ Deutlicher differenzierten schließlich Bundesratsmitglieder im 1971 beginnenden Heimgesetzverfahren: Wer etwa im Altenwohnheim lebte war nicht nur faktisch jünger, sondern wurde als Bewohner einer solchen auf eigene Haushaltsführung und Selbständigkeit setzenden Institution für weniger schutzbedürftig gehalten. ${ }^{124}$ Der Schutz, den heimgesetzliche Vorschriften vor allem für Hochaltrige in der vierten Lebensphase entfalten sollten, deutete sich in diesen Erwägungen zumindest schon an. Das 1974 an die Stelle des $\$ 38$ GewO tretende Heimgesetz hätte seinen Schwerpunkten nach ebenso gut Altenheimgesetz genannt werden können. Die darin enthaltene Mitwirkungsmöglichkeit wurde auf alle Heimbewohner erstreckt, obwohl Alter und Krankheit bereits in diesem Gesetzgebungsverfahren die Frage aufwerfen ließen, inwieweit eine Bewoh-

120 Zum Anliegen des Zweiten Betreuungsrechtsänderungsgesetzes (BGBl. I 2005, S. 1073) die „Eingriffe in das Selbstbestimmungsrecht auf das Notwendige zu beschränken“, Andreas Jurgeleit, Die Reform des Betreuungsrechts - Eine Übersicht, FGPrax 2005, S. 139-141. Kritisch gegenüber der Umsetzung der Reform, Wolf Crefeld, Betreuungsgesetz droht zu scheitern, in: Blätter der Wohlfahrtspflege 2007, S. $108 \mathrm{ff}$.

121 So etwa der Schweizer Gerontologe Adolf Lukas Vischer, in: Gesamtbericht Fürsorgetag 1959, S. 85. Der „ältere“ Mensch war außerdem stets der hilflose, schutzbedürftige: So zum Beispiel im Ergebnis der Bestandsaufnahme der Altenheime und Altenpflegeheime in Niedersachsen (1966), PA V/133 Dok. 28.

122 Hans-Joachim von Kondratowitz, Das ungeliebte Heim. Historische Gründe und neue Alternativen, in: Andreas Kruse/Ursula Lehr/Frank Oswald/Christoph Roll (Hrsg.), Gerontologie. Wissenschaftliche Erkenntnisse und Folgerungen für die Praxis, München 1988, S. 442.

123 BR-Drs. 593/1/65, S. 3.

124 BR-Drs. 173/72 (Beschluss), S. 20. 
nerbeteiligung zu verwirklichen war. ${ }^{125}$ In der in den Achtzigerjahren steigenden Zahl von Veröffentlichungen zum „Modethema“ Alter wurde das Altersbild zunehmend differenzierter. ${ }^{126}$ Pflege- und Hilfsbedürftigkeit traten als Charakteristika, die meist das Altern gegen Ende des Lebens prägten, vermehrt in den Vordergrund. ${ }^{127}$ So hob ein erster Familienbericht zur „Situation der älteren Menschen in der Familie“ 1986 zwar hervor, dass ein hohes Alter „nicht mehr notwendig mit dem Abbau und Verlust von Fähigkeiten verbunden " sei. ${ }^{128}$ Als erste Leitidee formulierten die Sachverständigen allerdings das selbstverantwortete Leben bei „Krankheit und Behinderung sowie in einer Pflegesituation“. ${ }^{129}$ Die Diskussionen um das erste Heimänderungsgesetz wurden entsprechend nicht mehr von Fragen des Dritten Alters, ${ }^{130}$ sondern von Hochaltrigkeit und Pflege dominiert. ${ }^{131}$ Die Tatsache, dass das Durchschnittsalter in Einrichtungen 80 bis 85 Jahre erreicht hatte, konnte gar nicht oft genug erwähnt werden. ${ }^{132}$ Die wachsende Anzahl von Bewohnern, die zur aktiven Mitwirkung nicht mehr in der Lage waren, sollte künftig ein von der konservativliberalen Koalition in das Gesetz eingeführter Heimfürsprecher vertreten. Detaillierte Kündigungsvorgaben für den Fall eintretender Pflegebedürftigkeit stellten faktisch einen Schutz des hohen Alters dar - wurde die Gefahr des sich verschlechternden Gesundheitszustands doch vor allem mit dem steigenden Alter im Laufe des Heimaufenthalts begründet. ${ }^{133}$ In der etwa zeitgleich mit dem Gesetzgebungsverfahren einsetzenden Altenberichterstattung der Bundesregierung spielte Hochaltrigkeit eine ähnlich indirekte Rolle ${ }^{134}$ und auch im Heimrecht setzte sich diese zunächst fort: Mit der Anwendung heimgesetzlicher Vorschriften auf die Kurzzeitpflege erstreckte der Gesetzgeber 1997 die bestehende Aufsicht auf Einrichtungen, die von pflegenden

125 Vgl. den Beitrag von Herbert Christ (FDP), BT Plenarprotokolle, 106. Sitzung, 7. Wp. (1972) 11.7.1974, S. 7227.

126 Birgit Baumgartl, Altersbilder und Altenhilfe. Zum Wandel der Leitbilder von Altenhilfe seit 1950, Opladen 1997, S. 175.

127 Birgit Baumgartl, Altersbilder und Altenhilfe. Zum Wandel der Leitbilder von Altenhilfe seit 1950, Opladen 1997, S. 208. Dass sich die Politik zunehmend mit dem hohen Alter beschäftigte zeigt u.a. die Kleine Anfrage der SPD Fraktion zu Lebensumständen „älterer und hochbetagter Frauen in der Bundesrepublik“ vom 31.7.1984, BT-Drs. 10/1738.

128 Vierter Familienbericht, BT-Drs. 10/6145, S. V.

129 BT-Drs. 10/6145, S. 178; Baumgartl hebt hervor, dass Maßnahmen zur Absicherung des Pflegerisikos an erster Stelle des Berichts genannt werden und der Bericht damit eine Punkt markiere, ,an dem sich die Problemsicht bezüglich Alter umzukehren beginnt.“, Baumgartl, Altersbilder, S. 181.

130 Zum Begriff des „Dritten Alters“ Peter Laslett, Das Dritte Alter: Historische Soziologie des Alters. Aus dem Englischen übersetzt und mit einer Einführung von Axel Flügel, Weinheim u.a. 1995. Zu Aktivitäten für Senioren in der zweiten Hälfte der Siebzigerjahre vgl. etwa die Rubrik „Senioren in Aktion“ im Dezemberheft der Blätter der Wohlfahrtspflege 1975.

131 Z.B. Erste Lesung, BT Plenarprotokoll, 162. Sitzung, 11. Wp. (1987) 29.9.1989, S. 12323 (C).

132 U.a. in: BT Plenarprotokoll, 202. Sitzung, 11. Wp. (1987) 15.3.1990, S. 15638.

133 Zum Heimänderungsgesetz: Thomas Klie, Zweiter Versuch, in: Altenpflege 1988, S. 197-201.

134 Bereits die Bundestagsdebatte zum ersten, noch von Bundesfamilienministerin Lehr initiierten Bericht wandte sich zum Beispiel dem Thema der Pflege und damit auch dem Vierten Alter zu, vgl. etwa die Rede von Erika Reinhardt (CDU/CSU), BT Plenarprotokoll, 211. Sitzung, 25.2.1994, S. 18299. 
Angehörigen genutzt werden konnte, ${ }^{135}$ und regulierte damit eine Institution, die sich tatsächlich in erster Linie an Hochbetagte und ihre Familien wandte. ${ }^{136}$ Als „Bevölkerungsgruppe" traten Hochaltrige erst im 21. Jahrhundert in Erscheinung. So widmete sich der Vierte Altenbericht der Bundesregierung 2002 ausdrücklich den über 80-jährigen anstatt einen Ausschnitt aus Lebensverhältnissen aller älterer Menschen zu thematisieren. ${ }^{137}$ Einige der neuen Ländergesetze nehmen sogar ausdrücklich auf das Alter Bezug ${ }^{138}$ und unterscheiden sich damit auch heute noch deutlich von dem, was das Zivilrecht in Gestalt des Familienrechts für das hohe Alter leistet.

Im Pflegschaftsrecht wurde auf das hohe Alter scheinbar keine Rücksicht genommen. Zwar war Hochbetagtheit sowohl in der Entwicklung des BGB vor der Jahrhundertwende als auch im „Palandt“ der Fünfzigerjahre als Tatbestand einer Gebrechlichkeitspflegschaft erwähnt. ${ }^{139}$ Nur vereinzelt legten gerichtliche Entscheidungen jedoch die hinter einer Pflegschaft stehende Altersgebrechlichkeit offen. Und noch seltener finden sich Beiträge, die aufzeigten, dass gerade ältere Menschen zahlenmäBig in besonderem Grad betroffen waren. ${ }^{140}$ Die Wirkung, die das Pflegschaftsrecht im Alter entfaltete, wurde erst mit der in den Achtzigerjahren beginnenden Reformdiskussion deutlich sichtbar. Die Debatten um die mit dem Betreuungsrecht einzuführende „Milieuanhörung“, die „unterbringungsähnlichen Maßnahmen“ sowie die „Altersvorsorgevollmacht“ kreisten dabei nicht mehr um das Alter allgemein. Das Dritte Alter blieb insofern außen vor, als eine rechtliche Betreuung aufgrund „seelischer Behinderung"141 an den Gesundheitszustand anknüpfte. Zwar sollte die Vollmacht vor dem Eintritt in das Vierte Alter vorbereitet sein. Die Wirkung der Vorsorge sowie der die genannten Problemkreise tangierenden Vorschriften diskutierten Juristen, Bundesrats- und Bundestagsmitglieder sowie die in den Gesetzgebungsprozess

135 So zumindest regelmäßig die Empfehlung, z.B. bei Gisela Mötzing/Susanna Schwarz, Leitfaden Altenpflege, München 2010, S. 69.

136 Vgl. etwa die Statistik aus Berlin (2003), aus der sich ergibt, dass sich alle von insgesamt 24 Kurzzeitpflegeheimen an ältere Menschen richten, während für Behinderte kein einziges spezifisch zur Verfügung stand, http://www.statistik-berlin.de/statistiken/sozialleistungen/i-pfle-5.htm (17.2.2011).

137 Stellungnahme der Bundesregierung zum Bericht der Sachverständigenkommission für den Vierten Altenbericht „Risiken, Lebensqualität und Versorgung Hochaltriger unter besonderer Berücksichtigung demenzieller Erkrankungen", S. 17, http://www.bmfsfj.de/bmfsfj/generator/BMFSFJ/Service/ Publikationen/publikationsliste, did=5362.html (25.1.2012).

138 Z. B. das Heimgesetz für Baden-Württemberg, in dem es in $\$ 1$ heißt: „, Dieses Gesetz gilt für Heime. Heime [...] sind Einrichtungen, die dem Zweck dienen, ältere Menschen [...] aufzunehmen, http:// www.biva.de/index.php?id=639 (17.2.2011).

139 Palandt. Bürgerliches Gesetzbuch, 17. Aufl. München u.a. 1958, \$1910, S. 1384.

140 Gerhard Krüger, Gefahrenpunkte bei der Anwendung des Hessischen Freiheitsentziehungsgesetzes, in: NDV 1954, S. 139-142, S. 141; W. Müller, Pflegschaften für alte Menschen, in: Blätter der Wohlfahrtspflege 1958, S. 395-396, S. 395.

141 Unter diesen Begriff waren altersspezifische Gebrechen zu subsumieren, BT-Drs. 11/4528 vom 11.5.1989, S. 116 . 
einbezogenen Experten ${ }^{142}$ aber in erster Linie anhand der spezifischen Situation Hochbetagter.

\section{Viertes Alter und Recht?}

Heimrecht und Betreuungsrecht sind demnach vor dem Hintergrund bestimmter Vorstellungen vom Alter und unter Einfluss zeitgenössischer Altersbilder geschaffen worden. Die Normen an sich blieben aber altersneutral formuliert. Niemand würde in Frage stellen, dass die Vorschriften auch für Pflegeheime gelten, in denen sich junge Erwachsene aufhalten. Ebenso schließen sie Betreute ein, die aufgrund ihrer nichtaltersspezifischen Krankheiten eines rechtlichen Beistandes bedürfen. Insofern ist es kaum verwunderlich, dass eine Annäherung von Alternsfragen und rechtlichen Problemen in der Rechtswissenschaft bis heute nur selten stattfindet. ${ }^{143}$ Auffallend ist, dass diese Beobachtung auch zu der Entwicklung von Alter und Recht in den beiden untersuchten Rechtsgebieten passt.

\section{Heimrecht}

Heimrecht zielte von seinen Anfängen her deutlich auf den Schutz von alten Menschen $\mathrm{ab}$ - Rechtswissenschaftler hatten mit heimgesetzlichen Vorschriften lange Zeit wenig zu tun. Die Ergänzung der Gewerbeordnung wurde maßgeblich durch den Altenhilfeausschuss des Deutschen Vereins angestoßen, in dem sich Personen verschiedener Fachrichtungen trafen. ${ }^{144}$ In die ersten Anhörungen zum Heimgesetz in Bundesrat und Bundestag waren Verwaltungsfachleute der Länderebene, Vertreter der Wohlfahrtsverbände und Heimleiter geladen, um einen Überblick über die Fragen des Heimrechts zu geben. Darunter mögen Juristen gewesen sein. Rechtswissenschaftler wurden jedoch erst im Rahmen der ersten Reform des Heimgesetzes konsultiert. $^{145}$

\section{Betreuungsrecht}

Ein ähnliches Bild der verzögerten Beschäftigung von Rechtswissenschaftlern mit altersspezifischen Rechtsfragen zeigen noch deutlicher das Pflegschafts- und Betreuungsrecht. Die Verwaltung, etwa in Berlin, thematisierte schon 1965 den steigenden Bedarf für Pfleger von Hochbetagten. ${ }^{146}$ Juristische Beiträge, die sich mit dem Zu-

142 Im Bundesrat dazu insb. die Sitzung des Unterausschuss des Rechtsausschusses Niederschrift über die Sitzung des UA des RA am 14.2.1989, S. 29 f., PA XI/294 A 2 Dok. 12; im Bundestag die Anhörung im Rechtsausschuss Stenographisches Protokoll (Teil I) der Sitzung des RA am 15.11.1989, PA XI/ 294 A 3 Dok. 39.

143 Eine Beschäftigung damit in der Gegenwart mahnt Gisela Zenz seit Langem immer wieder an, u.a. Gisela Zenz, Hilfebedarf und Persönlichkeitsrechte im Alter. Anforderungen an das Familienrecht, in: KritV 2010, S. 281-289.

144 Der Ausschuss wurde 1951 gegründet und sollte sich umfassend mit Altern und alten Menschen befassen, Fachausschuß für Fragen der Altenpflege und -fürsorge, in: NDV 1952, S. 57-58.

145 Anhörung vor dem Bundestag, Wortprot. des JFFGA am 15.11.1989, PA XI/224 A Dok. 21.

146 Pflegschaften, Beistandschaft, Vormundschaften/Bln., in: Soziale Arbeit 1966, S. 447 f. 
sammenspiel von Alter und Pflegschaft beschäftigten, finden sich aus diesem Zeitraum kaum. Rechtswissenschaftler beschäftigten vorrangig die dogmatischen Fragen, die sich aus der Rechtsprechung von Reichsgericht und Bundesgerichtshof ergaben. Eine Ausnahme bildete der Familienrechtler Joachim Gernhuber, der seit Mitte der Siebzigerjahre das Verhältnis zwischen Eltern und ihren Kindern als Pfleger und die damit einhergehenden generationellen und alternsspezifischen Probleme beleuchtete. ${ }^{147}$ Eine umfassende Wende erfuhr das Thema schließlich in den Arbeiten der Psychiatrie-Enquete: Die Kommissionsmitglieder räumten den psychiatrischen Problemen alter Menscher, gemessen an der noch relativ geringen öffentlichen Aufmerksamkeit, einen geradezu verblüffend hohen Stellenwert ein. ${ }^{148}$ Erst mit dem Vormundschaftsgerichtstag ${ }^{149}$ und dem Deutschen Juristentag ${ }^{150}$ nahmen sich aber schließlich Juristen in größerer Zahl explizit des rechtlichen Beistands für hochbetagte Menschen an. Im Zuge der Reformen des Pflegschaftsrechts etablierten sich hochbetagte, altersgebrechliche bzw. unter Altersdemenz leidende Menschen schließlich als eine Gruppe von Betroffenen. ${ }^{151}$ Zwar wurde um die Jahrtausendwende weiterhin beklagt, dass Probleme alter Menschen seitens der Familienrechtswissenschaft zu wenig Raum erhalten. ${ }^{152}$ Dennoch finden das Betreuungsrecht und die Frage der Autonomie Hochbetagter heute zunehmend auch von Juristen Beachtung. ${ }^{153}$

\section{Schluss}

Diese Beobachtungen zeigen, dass die quantitative Zunahme und qualitative Veränderung der Normen in beiden Rechtsgebieten seit den Sechzigerjahren in erster Linie das Alter erfasst hat. Dabei entstanden Vorschriften, von denen vor allem Betroffene im Vierten Lebensalter profitieren sollten. Mit ausgeprägten Vorgaben zum Verfahrensrecht verbesserte die Rechtsprechung die Rechtstellung von Pflegebefohlenen, unter denen sich auch in den Sechzigerjahren zahlreiche hochaltrige Menschen be-

147 Joachim Gernhuber, Der Senior und sein Zwangsvermögenspfleger, in: FamRZ 1976, S. 189-197.

148 Der Antrag der Abgeordneten zur Enquete umfasste „Psychisch Alterskranke“, BT-Drs. 6/474 vom 5.3.1970, Materialsammlung I, S. 3; außerdem gab es eine eigene Arbeitsgruppe „Gerontopsychiatrie“, Zwischenbericht BT-Drs. 7/1124, vom 19.10.1973, S. 15.

149 Vormundschaftsgerichtstag. Materialien und Ergebnisse des 1. Vormundschaftsgerichtstags vom 26. bis 29. Oktober 1988 in Bad Bevensen, München 1989.

150 Verhandlungen des siebenundfünfzigsten Deutschen Juristentags, München 1988.

151 Der Fachausschuss Altenhilfe stellte z. B. fest, dass Alter in der Stellungnahme des Deutschen Vereins zum Gesetzentwurf nicht ausreichend berücksichtigt wurde, Margarete Heinz, Fachausschuss V Altenhilfe (Sitzung am 27.2.1986), in: NDV 1986, S. 251. Zu alten Menschen als „Zielgruppe“: Albrecht Dieckmann, Empfiehlt es sich, das Entmündigungsrecht, das Recht der Vormundschaft und der Pflegschaft über Erwachsene sowie das Unterbringungsrecht neu zu ordnen?, in: JZ 1988, S. 789-800.

152 Gisela Zenz, Autonomie und Familie im Alter - (k)ein Thema für die Familienrechtswissenschaft?, in: Dieter Simon/Manfred Weiss (Hrsg.), Zur Autonomie des Individuums, Baden-Baden 2000, S. 483-508.

153 Markus Roth, Die Rechtsgeschäftslehre im demographischen Wandel. Stärkung der Autonomie sowie Schutzkonzepte bei Älteren und Minderjährigen, in: AcP 2008, S. 451-489; außerdem eine Reihe von Beiträgen in: KritV 2004. 
fanden. Mit dem Heimrecht begann der Gesetzgeber Einrichtungen zu regulieren und mit dem Betreuungsrecht eine Institution zu schaffen, die in erster Linie die Lebensbedingungen hochaltriger Menschen bestimmten. Im Rahmen dieser Verrechtlichung fand ein in der Lebenswirklichkeit existierender „Alter(n)sverlauf“ auch im Recht einen Niederschlag. Ein unterschiedliches Schutzbedürfnis alter Menschen, das sowohl an Wohnformen als auch an verschiedenen Graden von Eigenständigkeit festgemacht werden konnte, zeichnete sich in den Vorschriften ab. Hinter den Normen und Einzelfallentscheidungen standen bestimmte Vorstellungen eines aktiven oder hilfsbedürftigen Lebensalters und unterschiedlicher Bedürfnisse älterer Menschen. Welche Bedeutung diesen Vorstellungen zugemessen werden muss, zeigt sich unter anderem darin, dass der jüngste Altenbericht der Bundesregierung sich eigens dem Thema der Altersbilder widmet. ${ }^{154}$ Dieses bleibt zumindest so lange aktuell, wie auch optimistische Deutungen des Älterwerdens die Gefahr einer zunehmenden Abhängigkeit nicht hinwegreden können. ${ }^{155}$ Die auf den Altersbildern beruhenden Vorschriften tragen so zu der eingangs erwähnten Stabilität des Lebenslaufsmodells bei.

Die Lebenswelten Hochbetagter, sei es im Heim oder im eigenen Haushalt, wurden in der Bundesrepublik dann einer rechtlichen Kontrolle unterworfen, wenn die Hochaltrigen zunehmend auf Dritte angewiesen waren. Dabei hat sich die Perspektive des Rechts auf Alter und Altern im Laufe der Zeit ebenso stark verändert, wie sich der Blick von Kommunalverwaltung oder Sozialwissenschaft auf das Alter verschoben hat. ${ }^{156}$ In den Siebzigerjahren stand sowohl in der Heimgesetzgebung als auch in den ersten Reformdiskussionen um das Vormundschafts- und Pflegschaftsrechts im Rahmen der Psychiatrie-Enquete die Mitbestimmung des Aktiven im Vordergrund. Inzwischen hat sich der rechtliche Zugriff auf Einrichtungen und Institutionen, die Hochaltrige betreffen, demgegenüber zu einer Frage des Minimums an Selbstbestimmung für hochaltrige Pflegebedürftige und der Sicherung dieses Rechts gewandelt. ${ }^{157}$ Rechtlich wird sich an die Lebensphase des hohen Alters dabei immer noch weniger herangewagt, als die Präsenz des Themas in der Öffentlichkeit es vermuten lassen würde.

\section{Resumée (Stefan Ruppert)}

Die beiden rechtshistorischen Beispiele haben gezeigt, dass die juristische Strukturierung unseres Lebenslaufs weit über die Dreiteilung in Jugend, Erwerbsbiographie

154 Sechster Altenbericht der Bundesregierung, BT-Drs. 17/3185.

155 Paul Baltes urteilte: „Der Negativeffekt des hohen Alters ist um ein Vielfaches größer als der historische Fortschritt in der Vitalität des Alters“, Oma muss ran, in: DIE ZEIT vom 19.5.2005, Nr. 21.

156 Dazu umfassend die Studie von Birgit Baumgartl, Altersbilder und Altenhilfe. Zum Wandel der Leitbilder von Altenhilfe seit 1950, Opladen 1997.

157 Dies zeigt sich auch an den Versuchen seit dem Dritten Heimrechtsänderungsgesetz (BGBl. I 2001, S. 2970), Modelle des betreuten Wohnens, die möglicherweise mehr Handlungsspielraum als das konventionelle Pflegeheim bilden, vom Anwendungsbereich des Heimgesetzes auszunehmen. 
und Ruhestand hinausgeht. Gerade der Beginn und das Ende des menschlichen Standardlebenslaufs sind Gegenstand einer fortschreitenden Verrechtlichung. Setzte diese im Fall der Kleinkinder bemerkenswert früh bereits im 19. Jahrhundert ein, so ist die Verrechtlichung des Vierten Lebensalters im Wesentlichen erst eine Entwicklung der Bundesrepublik. An den beiden Beispielen ist auch deutlich geworden, dass die Verrechtlichung von Lebensphasen mit unterschiedlichen Regelungsinstrumenten vollzogen werden kann. Die Kleinkindphase ist durch Altersgrenzen strukturiert, das Vierte Lebensalter ist nicht an Altersgrenzen geknüpft. Der Gesetzgeber hatte beim Recht der Pflegeversicherung, dem Heim- oder Betreuungsrecht eben vor allem die Lebenssituation alter Menschen vor Augen. Entsprechend werden deren spezifische Lebenssituationen normativ gefasst. Die immer wieder thematisierte Destandardisierung von Lebensläufen spielgelt sich im normativen Lebenslauf nicht wider. Gerade weil Teillebensphasen wie die Kleinkindphase und die Schulzeit innerhalb der Jugend rechtlich gegeneinander abgegrenzt werden, ergeben sich erhebliche Schwierigkeiten, wenn man etwa die frühere Beendigung der Schulzeit durch eine frühere Einschulung erreichen will. Die Regelungsform der festen Altersgrenze ist ein vergleichsweise unflexibles Regelungsinstrument. Das liegt nicht nur daran, dass sie notwendig generalisiert und dem Einzelfall nicht immer gerecht werden kann. Ihre Veränderung stößt gesellschaftlich auch zumeist auf eine größere Gegenwehr wie etwa Debatten über die Veränderung des Wahlalters, des Renteneintrittsalters, aber auch der Schulpflicht zeigen. Dagegen ist die Beschreibung eines Altersstatus mittels biologisch-medizinischer und sozialer Kriterien, wie sie sich im Vierten Lebensalter findet, eindeutig flexibler.

Insgesamt lässt sich weiter eine Verrechtlichung des modernen Lebenslaufs beobachten. Welche Wechselwirkungen dieses Lebenslaufmodell mit der demographischen Struktur unserer Gesellschaft hat, ist wenig erforscht. Es dürfte aber deutlich geworden sein, dass die ausschließliche Betrachtung einer empirischen feststellbaren Lebenslaufstruktur ohne eine gleichzeitige Einbeziehung institutioneller und rechtlicher Rahmenbedingungen wenig aufschlussreich erscheint. 